\title{
Initial Development of Reduced-Order Models for Feedback Control of Axisymmetric Jets
}

\author{
Aniruddha Sinha*, Andrea Serrani ${ }^{\dagger}$ and Mo Samimy ${ }^{\ddagger}$ \\ The Ohio State University, Columbus, $\mathrm{OH}, 43235$
}

\begin{abstract}
Active control of high-speed and high Reynolds number axisymmetric jets for noise attenuation and bulk mixing enhancement is a topic of great current interest. An essential initial step in the implementation of feedback control to achieve the above goals is the development of a reduced-order model of the unforced jet. A number of modeling strategies are formulated hereby, and they are evaluated using an existing direct numerical simulation database of an unforced jet similar to our experimental configuration. A combination of proper orthogonal decomposition, spectral stochastic estimation, and Galerkin projection is used to derive the model from empirical data. Simulations of the reduced-order model are demonstrated to be sufficiently faithful to the full-order original simulation results to warrant its use for future control design.
\end{abstract}

\section{INTRODUCTION}

Jet noise has been a cause for concern since the commercialization of jet engine technology for civil and military aviation. In recent years, the problem has worsened with increasing number of flights, growth of residential population around airports, enactment of more stringent regulations, and deployment of significantly noisier high-performance military jets. Although jet noise is a mature research area with a history spanning more than five decades, there is still a lack of consensus on the fundamental mechanisms involved ${ }^{1}$. However, a common ground among the divergent viewpoints is the recognition of the importance of the large-scale coherent structures in the jet mixing layer, especially near the end of the potential core.

Apart from jet noise mitigation, research effort is also focused on enhancing bulk mixing in jets. Specifically, the hot gases exiting from the jet nozzle undergo bulk mixing with the ambient fluid in a process that ultimately leads to dissipation. It is of military interest to enhance this mixing so that the signature of the jet vanishes quickly. The rate of dissipation is clearly correlated with the dynamics of the large-scale structures in the jet mixing layer ${ }^{2}$.

From the above discussion, one can conclude that the disparate research fields of noise mitigation and bulk mixing enhancement in jets have the large-scale structures as a common denominator. Affecting the turbulence characteristics of flows by manipulating large-scale structures is within the realm of flow control, which is therefore appropriate for both these applications.

Localized arc filament plasma actuators (LAFPAs) have been developed and continuously improved for flow control applications at the Gas Dynamics and Turbulence Laboratory at The Ohio State University ${ }^{3}$. LAFPAs are capable of generating high-amplitude and high-bandwidth control signals, which are crucial actuator characteristics for controlling high-speed and high Reynolds number flows. These actuators provide intense but controlled localized Joule heating to manipulate the large-scale structures in the mixing layer by exciting the natural instabilities of the jet. Eight of these actuators have been deployed in a uniform azimuthal array at the periphery of the nozzle exit of Mach 0.9 and 1.3 axisymmetric jets. The effects of various forcing azimuthal modes and frequencies on the response of the mixing layer were investigated ${ }^{4,5}$. At particular forcing Strouhal numbers and azimuthal modes of operation, the length of the jet potential core was significantly reduced with an increase in the jet

\footnotetext{
*Graduate Student, Dept. of Mechanical Engineering.

Associate Professor, Dept. of Electrical and Computer Engineering.

The Howard D. Winbigler Professor of Engineering, Dept. of Mechanical Engineering, and Director, Gas Dynamics and Turbulence Laboratory, corresponding author (samimy.1@osu.edu)
} 
centerline velocity decay rate beyond the end of potential core. Plasma actuation was also shown to attenuate far-field noise, with reductions of 0.5 to over $1.0 \mathrm{~dB}$ over a range of forcing Strouhal numbers ${ }^{6,7}$. The above explorations were performed on unheated jets. Significant improvements in effectiveness of the LAFPAs have been observed in heated jets for both mixing enhancement ${ }^{8}$, and noise attenuation ${ }^{9}$. Open-loop forcing results demonstrated that the LAFPAs have significant control authority on high-speed and high Reynolds number jet flows for applications involving both noise attenuation and bulk-mixing enhancement.

The above investigations also showed that the optimal forcing parameters for a certain application may exist in a limited region in parameter-space and that the location of this region may be a function of the operating conditions - e.g. Mach number and temperature ratio. A common technique of rendering a system's performance relatively independent of operating conditions is to incorporate feedback. Closed-loop control of near-wall turbulence for drag-reduction, separation control over highlift devices, cylinder wake control, cavity tone suppression, etc., in low-speed and low Reynolds number flows have seen intense research activity in recent years. In comparison, the development of feedback control in high-speed and high Reynolds number free shear layers has not received as much attention. This is due to the lack of suitable actuators till recently, and the increased difficulty in modeling the highly turbulent flows of practical interest. The present work is the first step in an attempt to fill this void.

A simple yet robust form of closed-loop control is model-free feedback. Here, the system input-tooutput map is assumed to be static, and the controller is typically set up to vary the input to seek the maximum or minimum output in real-time. Several such controller algorithms have been successfully developed and implemented for the present application ${ }^{10}$. Although the simplicity of model-free controllers is attractive for flow control, their adaptation is generally slowed by the necessity of neglecting the system dynamics. Considerable improvements in performance may be realized with a model-based feedback controller, which is the most common paradigm in feedback control. The motivation for moving to a model-based approach is three-fold: ${ }^{11-13}(a)$ to gain a deeper understanding of the physics involved in the unforced jet, the forced jet, and the plasma actuation itself, $(b)$ to attain faster convergence to the optimal parameter regime than the case of model-free control, by incorporating a knowledge of the dynamics of the jet mixing layer in the model, and $(c)$ to achieve improvements in power consumption.

From the point of view of practical implementation, as well as the feasibility of actual design of the control law, it is essential that a small set of ODEs be able to approximately describe the dynamical behavior of the control system. However, the 'exact' dynamics of flows are governed by the infinitedimensional Navier-Stokes equation. Hence, any model-based flow control strategy must necessarily involve the development of a reduced-order model (ROM) of the flow. A number of methods have been employed to develop ROMs of flows of practical interest - they lend themselves to the following broad categorization.

1. Phenomenological models of flows are obtained by invoking intuitive arguments about the essential physics of the flow. Flows dominated by oscillations have been modeled in this manner in the past ${ }^{13-16}$. Unfortunately, the unforced high Reynolds number jet mixing layer is relatively disorganized and this makes the task of phenomenological modeling quite difficult.

2. Black-box modeling involves starting with an assumed structure of the ROM (number of dynamic states, degree of nonlinearity, form of the forcing term, etc.) before performing welldesigned experiments or simulations to identify the dynamical relation between the system input and output. Several implementations have been reported in the recent flow control literature ${ }^{17-20}$. This modeling approach does not incorporate much physical information about the flow, but may be the most viable option in particularly complex applications.

3. The Galerkin procedure involves two steps to arrive at the $\mathrm{ROM}^{21}$. In the first step, the kinematics of the flow are assumed to reside on a low-dimensional manifold, so that the infinite dimensional flow variables are represented by an expansion on a finite number of modes. This expansion is usually linear, but nonlinear expansions have been found to yield greater accuracy for certain flows in bounded domains ${ }^{22,23}$. In the next step, the dynamics of these modes are also assumed to reside on the same low-dimensional manifold, and this is enforced by the Galerkin projection (GP) of the Navier-Stokes equations onto this manifold. Depending on the origin of 
the expansion modes, Galerkin models have been categorized as mathematical, physical, or empirical ${ }^{15}$. Empirical Galerkin models, as the name suggests, derive their modes from experimental data or numerical simulations. They have been generally found to be the most accurate while employing the least number of $\operatorname{modes}^{15}$.

The most common technique of deriving modes from empirical data is the Proper Orthogonal Decomposition (POD) ${ }^{21,24}$. Its popularity stems from the fact that it is a linear procedure, and it objectively educes an orthogonal set of basis functions that optimally converges in the sense of a suitablydefined $\mathcal{L}_{2}$ norm of the projection error. The eduction of POD bases for axisymmetric jets was pioneered by Glauser et al. ${ }^{25}$ with several important contributions in recent years ${ }^{26-30}$.

Based on the above discussion of the available options for deriving ROMs, we decided to use POD and empirical GP for our feedback control application. POD-GP has been used for studying the dynamics of flows over the past 20 years ${ }^{31-36}$. In particular, in our lab, we have implemented a modelbased feedback controller for reducing cavity tones using this modeling strategy ${ }^{12,37,38}$.

An essential and non-trivial initial step in the design of a model-based feedback controller is the modeling of the unforced flow. The POD-GP procedure can result in ROMs with widely differing fidelity depending on the simplifying assumptions that are made. The choices made at this point will affect the later development of the actuated model. The present article delves into the details of modeling the pertinent near-field mixing layer of the unforced axisymmetric jet. The incorporation of the effect of actuation is deferred to a subsequent work.

A very useful (and, in fact, indispensable) tool for evaluating the various modeling strategies is a time-and spatially-resolved 3D volumetric database of the axisymmetric jet. Freund ${ }^{39}$ has performed a direct numerical simulation (DNS) of an unforced Mach 0.9 axisymmetric jet with Reynolds number based on jet diameter $(R e)$ of 3600. In spite of its low Reynolds number, most of its general characteristics, and especially the nature of its large-scale structures, were found to be similar to those of a Mach 1.3 jet with an Re of $1.06 \times 10^{6}$ in an experiment ${ }^{40}$. Thus, in this article, we employed this DNS database to assess various strategies for developing an ROM of this unforced axisymmetric jet. However, one should keep in mind that the ROM would be ultimately derived from experiments on the high Reynolds number jet, in unforced as well as forced operating conditions. This would be made explicit, for instance, when reference is made to the (virtual) particle image velocimetry (PIV) performed on the DNS database.

In Section 2, we provide a detailed discussion of the proposed strategy for building the ROM, while leaving various options open for later assessment. In Section 3, these strategies are applied to the DNS database, and the results of simulation of the various ROMs are employed to determine the most suitable modeling strategy. Concluding remarks are offered in Section 4.

\section{STEPS IN REDUCED-ORDER MODEL DEVELOPMENT}

Although experimental acquisition of velocity information over extended spatial domains has become quite common, it is still quite infeasible to simultaneously obtain density, pressure, and/or temperature fields in the same domain. Thus one is forced to make an incompressibility assumption in approximating the Navier-Stokes equations. This may be justified since a control-oriented model only needs to be good enough for representing the short-time-horizon dynamical relation between control inputs and sensor outputs ${ }^{41}$.

The flow domain of interest for ROM development is the mixing layer in the vicinity of the end of the potential core, as shown in Figure 1(a). In the axisymmetric jet, the azimuthal direction is homogenous and the radial direction is inhomogeneous. In the past, the axial direction was assumed to be homogenous, and Taylor's hypothesis was used to convert a time-resolved velocity measurement to an axially-resolved velocity database ${ }^{32}$. However, our preliminary experience with the DNS database indicated that such an assumption may not be correct for the current modeling purpose. Then, the empirical database for the ROM should consist of the 3-component 2-point velocity cross-correlation tensor with all possible pairs of axial and radial locations, and all possible azimuthal separations over the indicated $3 \mathrm{D}$ region. The acquisition of such a database is quite complicated. Another option is to collect snapshots of the 3-component velocity field over the 3D region. Although this information is not directly accessible in experiments, Tinney et al. ${ }^{29,30}$ presented an approximate technique for this purpose using spectral linear stochastic estimation (SLSE), and we would adopt this in our work. 


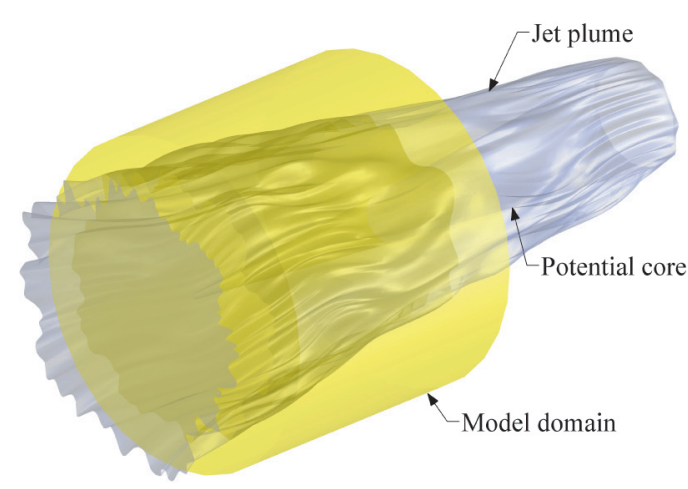

(a)

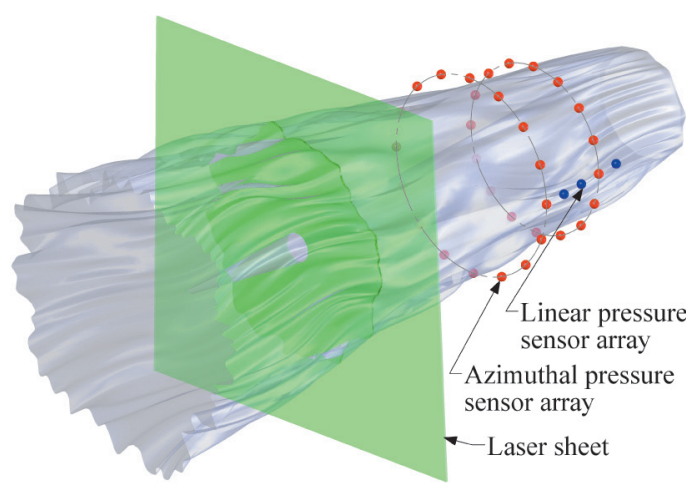

(b)

Figure 1. Schematic of axisymmetric jet. (a) Model domain. (b) Numerical experimental setup.

In the following sub-sections, we detail the modeling procedure. One of our contributions is to rigorously prove and enforce the symmetries of the axisymmetric flow. These symmetries $(a)$ augment the database collected from experiments, $(b)$ make computations easier, and $(c)$ model the flow more accurately ${ }^{42}$.

\subsection{Normalizations}

Let the jet exit velocity and nozzle exit diameter be $U_{\text {jet }}$ and $D$, respectively. All velocities and linear coordinates are implicitly normalized by these respective quantities. Time is normalized by the flow time scale $t^{+}:=D / U_{j e t}$. Pressure is normalized by $\rho_{j e t} U_{j e t}^{2}$, where $\rho_{j e t}$ is the ambient fluid density. With the kinematic viscosity of the ambient fluid denoted by $v_{j e t}$, the pertinent Reynolds number is $R e:=$ $U_{j e t} D / v_{j e t}$.

\subsection{D Proper Orthogonal Decomposition on Cross-Stream Slices}

The first step is the eduction of a low-dimensional basis for the velocity field over cross-stream slices of the axisymmetric jet mixing laye ${ }^{29}$ as shown in Figure l(b). The spatial domain is represented in cylindrical coordinates $(x, r, \theta)$, with the axial coordinate serving to parameterize the cross-stream slice location. The velocity vector is $\mathbf{V}:[0, R] \times \mathbb{T} \times \mathbb{R} \times \mathcal{X}_{v} \rightarrow \mathbb{R}^{3}, \mathbf{V}:(r, \theta, t ; x) \longmapsto\left(V_{x}, V_{r}, V_{\theta}\right)^{\mathrm{T}}$. Here, $R$ is the radial extent of the measurement domain, $\mathbb{T}$ is the circle group, $\mathbb{R}$ is the real line, and $\mathcal{X}_{v}$ is the set of axial locations of the cross-stream slices. The statistical stationarity and axisymmetry of the jet are used to define the mean velocity field as $\overline{\mathbf{V}}(r ; x):=E\left\{(1 / 2 \pi) \int_{-\pi}^{\pi} \mathbf{V}(r, \theta, t ; x) \mathrm{d} \theta\right\}$ Henceforth, unless otherwise mentioned, the expectation operator $E(\cdot)$ will signify the ensemble-average over all realizations indexed by $t$. Intuitively, $\overline{\boldsymbol{V}}_{\theta} \approx 0$, and this is enforced explicitly in the implementation. The fluctuating velocity vector is defined as $\mathbf{v}(r, \theta, t ; x):=\mathbf{V}(r, \theta, t ; x)-\overline{\boldsymbol{V}}(r ; x)$, with the three components being $v_{x}, v_{r}$, and $v_{\theta}$ respectively.

It is well-established ${ }^{24,43}$ that in the presence of a homogeneous and/or periodic direction in a flow, its POD devolves into the Fourier decomposition along that direction. The azimuthal Fourier transform of a function $f(\theta)$ will be denoted by $f(\theta) \stackrel{\mathcal{F}_{m}}{\longrightarrow} \hat{f}(m) ; \hat{f}(m):=(1 / 2 \pi) \int_{-\pi}^{\pi} f(\theta) \mathrm{e}^{-\mathrm{i} m \theta} \mathrm{d} \theta$. Here $m$ is the azimuthal mode, and $\mathrm{i}=\sqrt{-1}$. The inverse Fourier transform will be denoted by $\hat{f}(m) \stackrel{\mathcal{F}_{\theta}}{\longrightarrow} f(\theta) ; f(\theta)=\sum_{m=-\infty}^{\infty} \hat{f}(m) \mathrm{e}^{\mathrm{i} m \theta}$. With this, $\mathbf{v}$ is transformed as $\mathbf{v}(r, \theta, t ; x) \stackrel{F_{m}}{\longrightarrow} \hat{\mathbf{v}}(r, t ; x, m)$. Since $\mathbf{v}$ is real, $\hat{\mathbf{v}}$ is Hermitian in $m$.

The axisymmetry of the jet can be used to infer a symmetry condition. Consider an actual realization of $\mathbf{v}$ shown in Figure 2(a). The contour-map indicates the axial component, and the vector field is in the cross-stream plane. If enough realizations are collected in a perfectly axisymmetric jet, one should also expect to capture the simulated co-velocity field $\underline{\mathbf{v}}$ shown in Figure $2(\mathrm{~b})^{28}$. Of course, in an actual experiment, one cannot expect to collect both realizations. However, the collected database of 


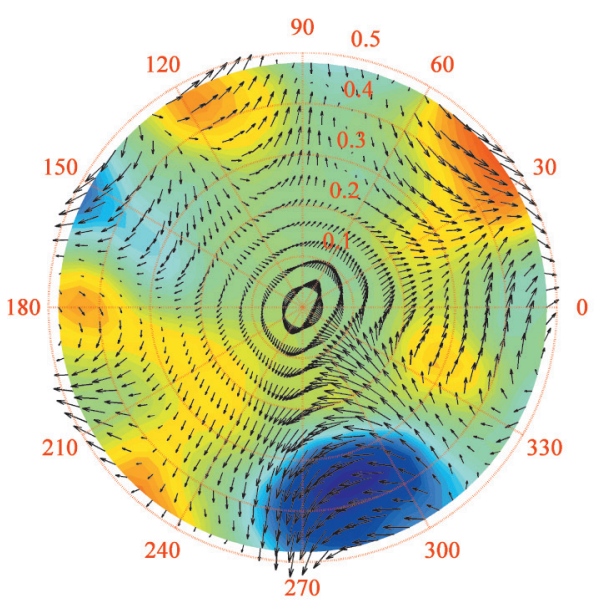

(a)

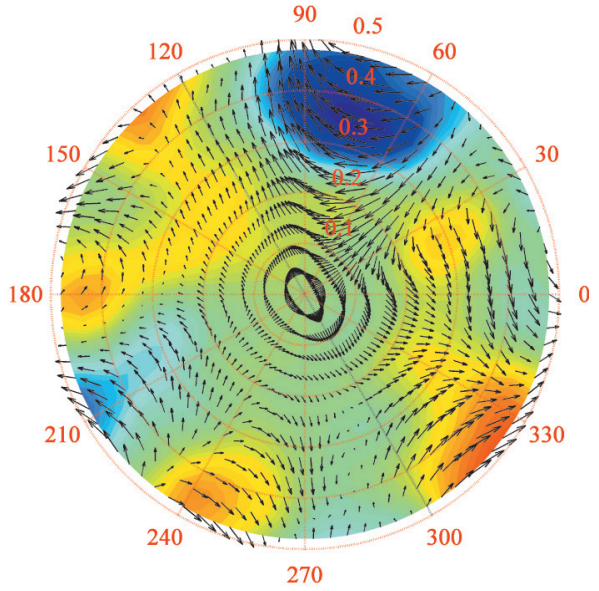

(b)

Figure 2. Enforcing axisymmetry. (a) Actual velocity field realization. (b) Corresponding co-velocity field.

realizations can be extended by appending the co-velocity field of each physical velocity field. To be precise, the two fields are related as follows:

$$
\underline{v}_{x}(r, \theta, t ; x):=v_{x}(r,-\theta, t ; x), \quad \underline{v}_{r}(r, \theta, t ; x):=\underline{v}_{r}(r,-\theta, t ; x), \quad \underline{v}_{\theta}(r, \theta, t ; x):=-v_{\theta}(r,-\theta, t ; x) .
$$

The two-point cross-correlation tensor is central to the POD; for all $i, j \in\{x, r, \theta\}$, it is defined as

$$
\Pi_{i j}\left(r, r^{\prime}, \vartheta ; x\right):=E\left[\frac{1}{2 \pi} \int_{-\pi}^{\pi} \frac{1}{2}\left\{v_{i}(r, \theta+\vartheta, t ; x) v_{j}\left(r^{\prime}, \theta, t ; x\right)+\underline{v}_{i}(r, \theta+\vartheta, t ; x) \underline{v}_{j}\left(r^{\prime}, \theta, t ; x\right)\right\} \mathrm{d} \theta\right]
$$

where we have used the azimuthal homogeneity of the axisymmetric jet. The relations in eqn (1) can be invoked to prove the following

$$
\begin{gathered}
\Pi_{i j}\left(r, r^{\prime}, \vartheta ; x\right)=\Pi_{i j}\left(r, r^{\prime},-\vartheta ; x\right), \quad \forall(i, j) \in\{(x, x),(r, r),(\theta, \theta),(x, r),(r, x)\}, \\
\Pi_{i j}\left(r, r^{\prime}, \vartheta ; x\right)=-\Pi_{i j}\left(r, r^{\prime},-\vartheta ; x\right), \quad \forall(i, j) \in\{(x, \theta),(r, \theta),(\theta, x),(\theta, r)\} .
\end{gathered}
$$

Such properties have indeed been verified in experiments ${ }^{27-29}$.

The azimuthal Fourier transform of $\prod_{i j}$ is defined in the usual manner: $\prod_{i j}\left(r, r^{\prime}, \vartheta ; x\right) \stackrel{\mathcal{F}_{m}}{\longrightarrow}$ $\hat{\Pi}_{i j}\left(r, r^{\prime}, x, m\right)$. Since $\prod_{i j}$ is real, $\hat{\Pi}_{i j}$ is Hermitian in $m$. The following can be deduced from eqn (3):

$$
\begin{aligned}
& \hat{\Pi}_{i j}\left(r, r^{\prime} ; x, m\right)=\mathcal{R}\left\{\hat{\Pi}_{i j}\left(r, r^{\prime} ; x, m\right)\right\}, \quad \forall(i, j) \in\{(x, x),(r, r),(\theta, \theta),(x, r),(r, x)\}, \\
& \hat{\Pi}_{i j}\left(r, r^{\prime} ; x, m\right)=\mathrm{i} \mathcal{I}\left\{\hat{\Pi}_{i j}\left(r, r^{\prime} ; x, m\right)\right\}, \quad \forall(i, j) \in\{(x, \theta),(r, \theta),(\theta, x),(\theta, r)\} .
\end{aligned}
$$

Here $\mathcal{R}(\cdot)$ and $\mathcal{I}(\cdot)$ respectively denote the real and imaginary parts of a complex quantity.

In practice, the following relation is used to compute $\hat{\prod}_{i j} 44$

$$
\hat{\Pi}_{i j}\left(r, r^{\prime} ; x, m\right)=E\left\{\hat{v}_{i}(r, t ; x, m) \hat{v}_{j}^{*}\left(r^{\prime}, t ; x, m\right)\right\}, \quad \forall i, j \in\{x, r, \theta\} .
$$

The asterisk denotes the adjoint operation, which reduces to the complex-conjugate transpose for our purpose. Subsequently, eqn (4) prompts the neglect of the real parts of the azimuthal shear stresses and the imaginary parts of the remaining correlation coefficients. In the past, analogous symmetries have been enforced for the kernel of a POD performed on a fully-developed channel flow $^{45}$.

Let $\hat{\mathbf{v}}^{(1)}$ and $\hat{\mathbf{v}}^{(2)}$ denote two fluctuating fields as above. The vector inner-product is defined as

$$
\left\langle\hat{\mathbf{v}}^{(1)}, \hat{\mathbf{v}}^{(2)}\right\rangle:=\int_{0}^{R} \hat{\mathbf{v}}^{(2)^{*}} \hat{\mathbf{v}}^{(1)} r \mathrm{~d} r .
$$


Then, for each $(x, m) \in \mathcal{X}_{v} \times(-\infty, \infty)$, the vector ID slice POD problem becomes the following integral eigenvalue problem ${ }^{26-29}$

$$
\int_{0}^{R} \sum_{j \in\{x, r, \theta\}} \hat{\Pi}_{i j}\left(r, r^{\prime} ; x, m\right) \hat{\phi}_{j}^{(n)}\left(r^{\prime} ; x, m\right) r^{\prime} \mathrm{d} r^{\prime}=\Xi^{(n)}(x, m) \hat{\phi}_{i}^{(n)}(r ; x, m), \quad \forall i \in\{x, r, \theta\}, \forall r \in[0, R] .
$$

The quantities $\Xi^{(n)}(x, m)$ and $\hat{\phi}_{i}^{(n)}(r ; x, m)$ are respectively the eigenvalue and the $i$ th component of the eigenfunction for the $n$th POD mode. Both are parameterized by the axial location of the cross-stream slice, and the azimuthal mode; the latter is also a function of the radial coordinate. The vectorial form of the eigenfunction is $\hat{\boldsymbol{\phi}}^{(n)}:=\left(\hat{\phi}_{x}^{(n)}, \hat{\phi}_{r}^{(n)}, \hat{\phi}_{\theta}^{(n)}\right)^{\mathrm{T}}$.

In the scalar POD, each velocity component is decomposed individually. The scalar inner product is defined for the $i$ th component of the velocity field as

$$
\left\langle\hat{v}_{i}^{(1)}, v_{i}^{(2)}\right\rangle:=\int_{0}^{R} \hat{v}_{i}^{(2)^{*}} \hat{v}_{i}^{(1)} r \mathrm{~d} r, \quad \forall i \in\{x, r, \theta\} .
$$

Then, for each $(x, m) \in \mathcal{X}_{v} \times(-\infty, \infty)$, the scalar ID slice-POD problem is

$$
\int_{0}^{R} \hat{\Pi}_{i i}\left(r, r^{\prime} ; x, m\right) \hat{\varphi}_{i}^{(n)}\left(r^{\prime} ; x, m\right) r^{\prime} \mathrm{d} r^{\prime}=\xi_{i}^{(n)}(x, m) \hat{\varphi}_{i}^{(n)}(r ; x, m), \quad \forall i \in\{x, r, \theta\}, \forall r \in[0, R] .
$$

Here the eigenfunctions $\hat{\varphi}$ are akin to those for the vector POD, but one obtains individual eigenvalues $\xi_{i}$ for the different components of velocity indexed by $i$. The vectorial form of the eigenfunction is $\hat{\varphi}^{(n)}=\left(\hat{\varphi}_{x}^{(n)}, \hat{\varphi}_{r}^{(n)}, \hat{\varphi}_{\theta}^{(n)}\right)^{\mathrm{T}}$. We will compare the results from the vector and scalar POD later; the reader is referred to other works that provide a more general discussion $28,29,36$.

The salient properties of the solutions of the POD problem have been discussed in depth elsewhere ${ }^{21,36}$. Here, the axisymmetry of the flow, captured in eqn (4), is invoked to note that the eigenfunctions of the vector 1D slice POD can always be normalized such that the following hold

$$
\hat{\phi}_{x}^{(n)}=\mathcal{R}\left\{\hat{\phi}_{x}^{(n)}\right\}, \quad \hat{\phi}_{r}^{(n)}=\mathcal{R}\left\{\hat{\phi}_{r}^{(n)}\right\}, \quad \hat{\phi}_{\theta}^{(n)}=\mathrm{i} \mathcal{I}\left\{\hat{\phi}_{\theta}^{(n)}\right\}, \quad \forall n .
$$

The solutions for the scalar POD problem satisfy identical relations. The kernels $\hat{\Pi}_{i j}$ of the POD problems are Hermitian in $m$; thus the eigensolutions are also Hermitian in $m$. Then, one can solve the eigenvalue problem for $m \geq 0$ only, and the results for $m<0$ can be recovered from the Hermitian property. The 1D POD problem is solved in the discrete radial domain. Typically the number of radial grid points are far fewer than the number of snapshots so that the original (or direct) POD method is employed ${ }^{24}$.

The properties of the POD allow one to exactly reconstruct any of the velocity field realizations that formed the original database, using a linear combination of all the eigenfunctions. However, it is typical to assume that any arbitrary velocity field can be approximately reconstructed using the first few (say, $N_{n, 1}$ ) eigenfunctions only ${ }^{21,24}$. For the vector 1D slice POD, we can write

$$
\hat{\mathbf{v}}(r, t, x, m) \approx \sum_{n=1}^{N_{n .1}} \hat{\beta}^{(n)}(t ; x, m) \hat{\boldsymbol{\phi}}^{(n)}(r ; x ; m), \quad \hat{\beta}^{(n)}(t ; x, m):=\left\langle\hat{\mathbf{v}}(r, t ; x, m), \hat{\boldsymbol{\phi}}^{(n)}(r ; x, m)\right\rangle,
$$

with $\hat{\beta}^{(n)}$ as the $n$th modal coefficient, which resolves the time information of the velocity field. Analogously, for each component $i \in\{x, r, \theta\}$, the scalar 1D slice POD allows the approximation

$$
\hat{v}_{i}(r, t ; x, m) \approx \sum_{n=1}^{N_{n \cdot 1}} \hat{\gamma}_{i}^{(n)}(t ; x, m) \hat{\varphi}_{i}^{(n)}(r ; x, m), \quad \hat{\gamma}_{i}^{(n)}(t ; x, m):=\left\langle\hat{v}_{i}(r, t ; x, m), \hat{\varphi}_{i}^{(n)}(r ; x, m)\right\rangle,
$$

where, $\hat{\gamma}_{i}^{(n)}$ is the $n$th modal coefficient for the $i$ th component of velocity. Since all of $\hat{\mathbf{v}}, \hat{\boldsymbol{\phi}}^{(n)}$, and $\hat{\varphi}_{i}^{(n)}$ are Hermitian in $m$, so are the modal coefficients $\hat{\beta}^{(n)}$ and $\hat{\gamma}_{i}^{(n)}$, for all $n$, and for all $i \in\{x, r, \theta\}$.

\subsection{D Velocity Field Reconstruction using Spectral Linear Stochastic Estimation}

Stochastic estimation was originally introduced to educe coherent structures in turbulent flows ${ }^{46}$. Subsequently, it has also been employed for estimating velocity fields using minimal measurements and a knowledge of the spatial correlations in the flow ${ }^{47}$. The original technique estimated the 
velocity directly ${ }^{47}$. In the classical version of the complementary technique, the estimated velocity field was projected onto the POD basis, and the projected field was deemed the final estimated field $^{48}$. Subsequently, the complementary technique was modified in two important ways ${ }^{49,50}$. The POD modal coefficients of the velocity field were estimated directly so that the estimated velocity field was reconstructed by weighting the POD basis by these coefficients. Additionally, a different physical quantity (eg. pressure, surface shear stress, etc.) was used as the unconditional variable. The concept was later extended by decomposing both the unconditional and conditional fields into their respective low-dimensional modes, be they Fourier or POD, before linking the corresponding modal coefficients through stochastic estimation ${ }^{30}$. The successive modifications were implemented to take advantage of the increased correlations between the low-dimensional quantities, thereby reducing computations without sacrificing (or, in some cases, actually improving) the accuracy of reconstruction. The spectral variant, SLSE, was implemented for statistically stationary flows, where the correlation was computed in the temporal Fourier domain ${ }^{30,51,52}$. This was shown to be especially useful whenever the spectral features of the conditional and unconditional variables were disparate, and/or significant time delays existed between them ${ }^{52}$. Both these effects are manifest in the present application.

In the modified complementary SLSE formulated by Tinney et al. ${ }^{30}$, time-resolved pressure measurements in the irrotational near-field of the axisymmetric jet were used as the unconditional variable to simultaneously reconstruct the conditional velocity field on several cross-stream slices through the jet mixing layer. It will soon be evident that this necessitates the computation of the crossspectral tensor between pressure and velocity. This was addressed by capturing stereo-PIV snapshots separately on each pertinent cross-stream slice, but at known instants of the pressure-record ${ }^{30}$. The original work relied on a single azimuthal array of pressure sensors, but the reconstruction can be made more accurate by adding an axial linear array of pressure sensors, at little extra cost. Figure 1(b) shows an even more general arrangement of pressure sensors that is used to formulate the problem. Consider $N_{p}^{a}$ azimuthal arrays of pressure transducers arranged at different axial locations $x \in \mathcal{X}_{p}^{a}$. In addition, suppose that there are $N_{p}^{l}$ individual pressure sensors at different axial locations $x \in \mathcal{X}_{p}^{l}$. that do not belong to any azimuthal array. Although the development does not need the individual sensors to form a linear array, they would be assumed to be in a straight line at $\theta=0$ for notational convenience. Without loss of generality, it is also assumed that all pressure sensors are located on the surface of a virtual cone co-axial with the jet, so that their radial locations are a function of their axial locations.

The individual pressure signals from the sensors in the azimuthal arrays are denoted as $P^{a}: \mathbb{T} \times \mathbb{R} \times$ $\mathcal{X}_{p}^{a} \rightarrow \mathbb{R}, P^{a}:(\theta, t ; x) \longmapsto \mathbb{R}$. As before, the mean and fluctuating pressure are defined as $\bar{P}^{a}(x):=$ $E\left\{(1 / 2 \pi) \int_{-\pi}^{\pi} P^{a}(\theta, t ; x) \mathrm{d} \theta\right\}$ and $p^{a}(\theta, t ; x):=P^{a}(\theta, t ; x)-\bar{P}^{a}(x)$, respectively. Similarly, the pressure signals on the linear array are denoted as $P^{l}: \mathbb{R} \times \mathcal{X}_{p}^{l} \rightarrow \mathbb{R}, P^{l}:(t ; x) \longmapsto \mathbb{R}$. The corresponding time-mean and fluctuating quantities are respectively $\bar{P}^{l}(x):=E\left\{P^{l}(t ; x)\right\}$, and $p^{l}(t ; x):=P^{l}(t ; x)-\bar{P}^{l}(x)$. The azimuthal Fourier transform of $p^{a}$ is defined in the usual manner: $p^{a}(\theta, t ; x) \stackrel{\mathcal{F} m}{\longrightarrow} \hat{p}^{a}(t ; x, m)$.

The formulation of the SLSE is quite similar for the three cases that are studied here (all are performed in the azimuthal Fourier domain): (a) the original technique for estimating the velocity field $\hat{\mathbf{v}}(r, t ; x$, $m)$, (b) the complementary technique for estimating the vector POD modal coefficients $\hat{\beta}^{(n)}(t ; x, m)$, and $(c)$ the complementary technique for estimating the scalar POD modal coefficients $\hat{\gamma}_{i}^{(n)}(t ; x, m), \forall i \in\{x, r, \theta\}$. We will show the equations for the last case, as those for the other two cases can be readily deduced from it.

The temporal finite Fourier transforms of the pressure signals are defined as ${ }^{44}$ :

$$
\check{\hat{p}}^{a}(x, m, f):=\int_{t=t_{0}-T / 2}^{t_{0}+T / 2} \hat{p}^{a}(t ; x, m) \mathrm{e}^{-2 \pi \mathrm{i} f t} \mathrm{~d} t, \quad \check{p}^{l}(x, f):=\int_{t=t_{0}-T / 2}^{t_{0}+T / 2} p^{l}(t ; x) \mathrm{e}^{-2 \pi \mathrm{i} f t} \mathrm{~d} t,
$$

where $f$ is the temporal frequency, and $t_{0}$ locates the mid-point of a time-series of length $T$. Let the temporal Fourier transform of $\hat{\gamma}_{i}^{(n)}\left(t ; x^{\prime}, m\right)$ be denoted as $\check{\hat{\gamma}}_{i}^{(n)}\left(x^{\prime}, m, f\right)$, where $x^{\prime} \in \mathcal{X}_{v}$ is the location of the crossstream slice. Generalizing the formulation of Tinney et $\mathrm{al}^{30}{ }^{30}$, and denoting the estimate of any quantity $w$ by $\widetilde{w}$ the modal coefficient $\check{\hat{\gamma}}_{i}^{(n)}\left(x^{\prime}, m, f\right)$ is estimated as 


$$
\tilde{\hat{\gamma}}_{i}^{(n)}\left(x^{\prime}, m, f\right):=\sum_{j=1}^{N_{p}^{a}} \mathcal{L}_{\gamma_{i}}^{u,(n)^{*}}\left(x_{j}, x^{\prime}, m, f\right) \check{\hat{p}}^{a}\left(x_{j}, m, f\right)+\sum_{k=1}^{N_{p}^{l}} \mathcal{L}_{\gamma_{i}}^{l,(n)^{*}}\left(x_{k}, x^{\prime}, m, f\right) \check{p}^{l}\left(x_{k}, f\right) .
$$

Einstein's summing convention should not be followed for $m$ and $f^{30}$. To minimize the estimation error $E\left\{\left[\tilde{\hat{\gamma}}_{i}^{(n)}\left(x^{\prime}, m, f\right)-\left.\check{\hat{\gamma}}_{i}^{(n)}\left(x^{\prime}, m, f\right)\right|^{2}\right\}\right.$ for each individual $x^{\prime}, i, n, m$, and $f$, the standard least-squares technique yields the following set of $N_{p}^{a}+N_{p}^{l}$ coupled linear equations for the estimation coefficients $\mathcal{L}$ :

$$
\mathbf{S}_{p p}(m, f) \mathcal{L}_{\gamma_{i}}^{(n)}\left(x^{\prime}, m, f\right)=\mathbf{S}_{p \gamma_{i}}^{(n)}\left(x^{\prime}, m, f\right),
$$

With

$$
\begin{aligned}
& \mathbf{S}_{p p}(m, f) \\
& :=\left[\begin{array}{cccccc}
S_{p p}^{a a}\left(x_{1}, x_{1}, m, f\right) & \cdots & S_{p p}^{a a}\left(x_{1}, x_{N_{p}^{a}}, m, f\right) & S_{p p}^{a l}\left(x_{1}, x_{1}, m, f\right) & \cdots & S_{p p}^{a l}\left(x_{1}, x_{N_{p}^{l}}, m, f\right) \\
\vdots & \ddots & \vdots & \vdots & \ddots & \vdots \\
S_{p p}^{a a}\left(x_{N_{p}^{a}}, x_{1}, m, f\right) & \cdots & S_{p p}^{a a}\left(x_{N_{p}^{a}}, x_{N_{p}^{a}}, m, f\right) & S_{p p}^{a l}\left(x_{N_{p}^{a}}, x_{1}, m, f\right) & \cdots & S_{p p}^{a l}\left(x_{N_{p}^{a}}, x_{N_{p}^{l}}, m, f\right) \\
S_{p p}^{a l^{*}}\left(x_{1}, x_{1}, m, f\right) & \cdots & S_{p p}^{a l^{*}}\left(x_{N_{p}^{a}}, x_{1}, m, f\right) & S_{p p}^{l l}\left(x_{1}, x_{1}, f\right) & \cdots & S_{p p}^{l l}\left(x_{1}, x_{N_{p}^{l}}, f\right) \\
\vdots & \ddots & \vdots & \vdots & \ddots & \vdots \\
S_{p p}^{a l^{*}}\left(x_{1}, x_{N_{p}^{l}}, m, f\right) & \cdots & S_{p p}^{a * *}\left(x_{N_{p}^{a}}, x_{N_{p}^{l}}, m, f\right) & S_{p p}^{l l}\left(x_{N_{p}^{l}}, x_{1}, f\right) & \cdots & S_{p p}^{l l}\left(x_{N_{p}^{l}}, x_{N_{p}^{l}}, f\right)
\end{array}\right], \\
& \mathcal{L}_{\gamma_{i}}^{(n)}\left(x^{\prime}, m, f\right) \\
& :=\left[\begin{array}{llllll}
\mathcal{L}_{\gamma_{i}}^{a,(n)}\left(x_{1}, x^{\prime}, m, f\right) & \cdots & \mathcal{L}_{\gamma_{i}}^{a,(n)}\left(x_{N_{p}^{a}}, x^{\prime}, m, f\right) & \mathcal{L}_{\gamma_{i}}^{l,(n)}\left(x_{1}, x^{\prime}, m, f\right) & \cdots & \mathcal{L}_{\gamma_{i}}^{l,(n)}\left(x_{N_{p}^{l}}, x^{\prime}, m, f\right)
\end{array}\right]^{\mathrm{T}}, \\
& \mathbf{S}_{p \gamma_{i}}^{(n)}\left(x^{\prime}, m, f\right) \\
& :=\left[\begin{array}{llllll}
S_{p \gamma_{i}}^{a,(n)}\left(x_{1}, x^{\prime}, m, f\right) & \cdots & S_{p \gamma_{i}}^{a,(n)}\left(x_{N_{p}^{a}}, x^{\prime}, m, f\right) & S_{p \gamma_{i}}^{l,(n)}\left(x_{1}, x^{\prime}, m, f\right) & \cdots & S_{p \gamma_{i}}^{l,(n)}\left(x_{N_{p}^{l}}, x^{\prime}, m, f\right)
\end{array}\right]^{\mathrm{T}} .
\end{aligned}
$$

Here, the cross-spectral tensors of pressure are defined as

$$
\begin{gathered}
S_{p p}^{a a}\left(x_{j}, x_{k}, m, f\right):=\lim _{T \rightarrow \infty} \frac{1}{T} E\left\{\check{\hat{p}}^{a}\left(x_{j}, m, f\right) \check{\hat{p}}^{a *}\left(x_{k}, m, f\right)\right\}, \quad \forall j, k \in\left[1, N_{p}^{a}\right] \\
S_{p p}^{a l}\left(x_{j}, x_{k}, m, f\right):=\lim _{T \rightarrow \infty} \frac{1}{T} E\left\{\check{\hat{p}}^{a}\left(x_{j}, m, f\right) \check{p}^{\prime *}\left(x_{k}, f\right)\right\}, \quad \forall j \in\left[1, N_{p}^{a}\right], \forall k \in\left[1, N_{p}^{l}\right] \\
S_{p p}^{l l}\left(x_{j}, x_{k}, f\right):=\lim _{T \rightarrow \infty} \frac{1}{T} E\left\{\check{p}^{l}\left(x_{j}, f\right) \check{p}^{l^{*}}\left(x_{k}, f\right)\right\}, \quad \forall j, k \in\left[1, N_{p}^{l}\right] .
\end{gathered}
$$

The expectation operator is the un-weighted average over different independent time records of length $T$. With the experimental realities in mind, the cross-spectral tensors between pressure and the modal coefficients are computed in a different, but equivalent, manner ${ }^{44}$ :

$$
\begin{aligned}
& S_{p \gamma_{i}}^{a,(n)}\left(x_{j}, x^{\prime}, m, f\right):=\int_{\tau=-\infty}^{\infty} E\left\{\hat{p}^{a}\left(t+\tau ; x_{j}, m\right) \hat{\gamma}_{i}^{(n)^{*}}\left(t ; x^{\prime}, m\right)\right\} \mathrm{e}^{-2 \pi \mathrm{if} \tau} \mathrm{d} \tau, \quad \forall j \in\left[1, N_{p}^{a}\right], \forall x^{\prime} \in \mathcal{X}_{v} \\
& S_{p \gamma_{i}}^{l,(n)}\left(x_{j}, x^{\prime}, m, f\right):=\int_{\tau=-\infty}^{\infty} E\left\{p^{l}\left(t+\tau ; x_{j}\right) \hat{\gamma}_{i}^{(n)^{*}}\left(t ; x^{\prime}, m\right)\right\} \mathrm{e}^{-2 \pi i j \tau} \mathrm{d} \tau, \quad \forall j \in\left[1, N_{p}^{l}\right], \forall x^{\prime} \in \mathcal{X}_{v} .
\end{aligned}
$$

Here, $t$ indicates the instant in the pressure record when the snapshot of the velocity field is captured on the cross-stream slice. Several such pairs of pressure records and velocity snapshots must be captured for convergence of the statistics; the expectation operator signifies an ensemble-average over all such 
pairs. The axisymmetry of the flow can be used to prove that cross-spectral tensors and the estimation coefficients are Hermitian functions of the temporal frequency $f$; these were enforced in our work.

Once $\stackrel{\times}{\gamma}_{i}^{(n)}\left(x^{\prime}, m, f\right)$ is estimated, inverse Fourier transform yields the estimate of $\hat{\gamma}_{i}^{(n)}\left(t ; x^{\prime}, m\right)$. This estimate is most accurate for $t=t_{0}$, the center of the original pressure time-series. So, it is best to perform the steps in eqns (13) and (14), and the inverse transform, separately for each time instant $t_{0}$ at which the velocity field is desired. Since the SLSE is intended for off-line implementation, accuracy considerations can be allowed to trump computational efficiency. The actual velocity field is reconstructed using eqn (12).

For Tinney et al. ${ }^{30}$, experimental expediency dictated that the cross-stream PIV slices could not be taken on an axial grid that was fine enough for the reliable computation of the required spatial derivatives. Cubic spline interpolation was used to solve this problem ${ }^{53,54}$. The same method is adopted here to render the database amenable for the subsequent GP. Along with the fluctuating velocity field, the GP also requires the spatially-resolved mean velocity field. This can be reconstructed by again using cubic spline interpolation to estimate the mean velocity on a grid of desired axial resolution from the measured mean velocities on the original coarse grid of cross-stream slices.

\subsection{D Proper Orthogonal Decomposition on 3D Velocity Database}

We have detailed various methods for obtaining a database consisting of snapshots of the 3 components of velocity on a 3D cylindrical region of the form shown in Figure 1(a). In the current work, one other possibility is to use the DNS database directly. To unify the notation, the spatially-resolved velocity field is now denoted by $\mathbf{U}:\left[X_{1}, X_{2}\right] \times[0, R] \times \mathbb{T} \times \mathbb{R} \rightarrow \mathbb{R}^{3}, \mathbf{U}:(x, r, \theta, t) \longmapsto\left(U_{x}, U_{r}, U_{\theta}\right)^{\mathrm{T}}$. Here, $X_{1}$ and $X_{2}$ denote the upstream and downstream bounds of the axial domain; note that $\left[X_{1}, X_{2}\right]$ should be covered by $\mathcal{X}_{v}$ for the reconstructed database detailed above. As before, the mean and fluctuating velocity fields are defined as $\overline{\mathbf{U}}(x, r):=E\left\{(1 / 2 \pi) \int_{-\pi}^{\pi} \mathbf{U}(x, r, \theta, t) \mathrm{d} \theta\right\}$ and $\mathbf{u}(x, r, \theta, t):=\mathbf{U}(x, r, \theta, t)-\overline{\mathbf{U}}(x, r)$, respectively.

The components of the fluctuating field are respectively $u_{x}, u_{r}$, and $u_{\theta}$. The azimuthal Fourier transform of the fluctuating velocity field is defined as $\mathbf{u}(x, r, \theta, t) \stackrel{\mathcal{F}_{m}}{\longrightarrow} \hat{\mathbf{u}}(x, r, t ; m)$.

The formulation of the 2D POD is very similar to the ID slice POD; the only difference is that the eigenfunctions cease to be parameterized by the axial location of the cross-stream slice, and instead become continuous functions of the axial coordinate. The basic symmetry condition established in eqn (1) still holds with the obvious modification, so that all the symmetries carry over. Therefore, we give a minimal description of the 2D POD, mainly to establish notation for later reference. Only the vector POD is pursued here since it produces superior results to the scalar version in the subsequent $\mathrm{GP}^{36}$.

Let $\hat{\mathbf{u}}^{(1)}$ and $\hat{\mathbf{u}}^{(2)}$ denote two fluctuating fields as above. The vector inner-product is defined as

$$
\left\langle\hat{\mathbf{u}}^{(1)}, \hat{\mathbf{u}}^{(2)}\right\rangle:=\int_{X_{1}}^{X_{2}} \int_{0}^{R} \hat{\mathbf{u}}^{(2)^{*}} \hat{\mathbf{u}}^{(1)} r \mathrm{~d} r \mathrm{~d} x
$$

Then, for each $m \in(-\infty, \infty)$, the vector 2D POD becomes the following integral eigenvalue problem

$$
\begin{aligned}
& \int_{X_{1}}^{X_{2}} \int_{0}^{R} \sum_{j \in\{x, r, \theta\}} E\left\{\hat{u}_{i}(x, r, t ; m) \hat{u}_{j}^{*}\left(x^{\prime}, r^{\prime}, t ; m\right)\right\} \hat{\Phi}_{j}^{(n)}\left(x^{\prime}, r^{\prime}, m\right) r^{\prime} \mathrm{d} r^{\prime} \mathrm{d} x^{\prime} \\
& =\Lambda^{(n)}(m) \hat{\Phi}_{i}^{(n)}(x, r ; m), \quad \forall i \in\{x, r, \theta\}, \forall(x, r) \in\left[X_{1}, X_{2}\right] \times[0, R] .
\end{aligned}
$$

The quantities $\Lambda^{(n)}(m)$ and $\hat{\Phi}_{i}^{(n)}(x, r ; m$, ) are respectively the eigenvalue and the $i$ th component of the eigenfunction for the $n$th POD mode. Both are parameterized by the azimuthal mode; the latter is also a function of the axial and radial coordinates. The vectorial form of the eigenfunction is

$$
\hat{\boldsymbol{\Phi}}^{(n)}:=\left(\hat{\Phi}_{x}^{(n)}, \hat{\Phi}_{r}^{(n)}, \hat{\Phi}_{\theta}^{(n)}\right)^{\mathrm{T}} \text {. }
$$

As an aside, the POD problem can be solved by the original method ${ }^{24}$ or the snapshot method ${ }^{42}$ depending on how the total number of points on the $2 \mathrm{D}$ grid compares to the number of realizations needed for statistical convergence. In the snapshot method, one does not explicitly use the kernel shown in eqn (19), so that its symmetries cannot be applied directly. Instead, an option is to extend the database of realizations with the co-velocity field as described before (use eqn (1), mutatis mutandis) ${ }^{42}$. Of course, this doubles the number of realizations, thereby making the snapshot method less attractive. 
Once the eigenfunctions are obtained, a low-dimensional reconstruction of the velocity field (in the Fourier azimuthal domain) can be obtained using only the first $N_{n, 2}$ eigenfunctions:

$$
\hat{\mathbf{u}}(x, r, t ; m) \approx \sum_{n=1}^{N_{n, 2}} \hat{\alpha}^{(n)}(t ; m) \hat{\boldsymbol{\Phi}}^{(n)}(x, r ; m), \hat{\alpha}^{(n)}(t ; m)=\left\langle\hat{\mathbf{u}}(x, r, t ; m), \hat{\boldsymbol{\Phi}}^{(n)}(x, r ; m)\right\rangle .
$$

As for the ID POD, since $\hat{\mathbf{u}}$ and $\hat{\boldsymbol{\Phi}}$ are Hermitian in $m$, so are the modal coefficients $\hat{\alpha}$. The subsequent GP models the dynamics of $\hat{\alpha}$, and their Hermitian nature means that the model only need consider the non-negative azimuthal modes. This reduces the size of the ROM by almost a half.

\subsection{Galerkin Projection}

The non-dimensionalized incompressible Navier-Stokes equations in cylindrical coordinates are ${ }^{55}$

$$
\begin{aligned}
\frac{\partial U_{i}}{\partial t}= & -(\mathbf{U} \cdot \nabla) U_{i}+\frac{U_{\theta}^{2}}{r} \delta_{i r}-\frac{U_{r} U_{\theta}}{r} \delta_{i \theta}-(\nabla P)_{i} \\
& +\frac{1}{R e}\left\{\nabla^{2} U_{i}+\left(-\frac{U_{r}}{r^{2}}-\frac{2}{r^{2}} \frac{\partial U_{\theta}}{\partial \theta}\right) \delta_{i r}+\left(-\frac{U_{\theta}}{r^{2}}+\frac{2}{r^{2}} \frac{\partial U_{r}}{\partial \theta}\right) \delta_{i \theta}\right\}, \quad i \in\{x, r, \theta\} .
\end{aligned}
$$

Here $P$ is the static pressure field, $\delta$ is the Kronecker delta, and $\nabla$ and $\nabla^{2}$ are respectively the gradient and Laplacian operators. In applying the Reynolds decomposition to the Navier-Stokes equations, a dichotomy is noticed in the ROM literature regarding the appropriate mean field representation. One group has used a steady mean field derived from empirical data by ensemble-averaging as well as averaging over any homogenous direction, if applicable $35,38,56-58$. Another group has used simplifying assumptions to adopt a slowly time-varying mean field averaged over all homogenous directions, followed by its representation in terms of the Reynolds stresses ${ }^{15,16,31-33}$. This is said to ensure bounded state trajectories by providing some feedback from the Reynolds stresses to the turbulence production mechanism (the gradient of the mean-field). For the axisymmetric jet, however, the underlying assumptions were not borne out by our investigations using the DNS database, thereby prompting the adoption of a steady empirical mean field.

Applying the ensemble- and azimuthal-averaging to eqn (21) and subtracting the result from the original, one obtains the dynamics of the velocity fluctuations as

$$
\begin{aligned}
\frac{\partial u_{i}}{\partial t}= & -\bar{U}_{x} \frac{\partial u_{i}}{\partial x}-\bar{U}_{r} \frac{\partial u_{i}}{\partial r}-u_{x} \frac{\partial \bar{U}_{x}}{\partial x} \delta_{i x}-u_{r} \frac{\partial \bar{U}_{x}}{\partial r} \delta_{i x}-u_{x} \frac{\partial \bar{U}_{r}}{\partial x} \delta_{i r}-u_{r} \frac{\partial \bar{U}_{r}}{\partial r} \delta_{i r}-\frac{\bar{U}_{r} u_{\theta}}{r} \delta_{i \theta} \\
& -(u \cdot \nabla) u_{i}+\overline{(u \cdot \nabla) u_{i}}+\frac{u_{\theta}^{2}-\overline{u_{\theta}^{2}}}{r} \delta_{i r}-\frac{u_{r} u_{\theta}-\overline{u_{r} u_{\theta}}}{r} \delta_{i \theta}-(\nabla p)_{i} \\
& +\frac{1}{\operatorname{Re}}\left\{\nabla^{2} u_{i}+\left(-\frac{u_{r}}{r^{2}}-\frac{2}{r^{2}} \frac{\partial u_{\theta}}{\partial \theta}\right) \delta_{i r}+\left(-\frac{u_{\theta}}{r^{2}}+\frac{2}{r^{2}} \frac{\partial u_{r}}{\partial \theta}\right) \delta_{i \theta}\right\}, \quad i \in\{x, r, \theta\} .
\end{aligned}
$$

Here, $p$ is the pressure fluctuation, with azimuthal Fourier transform defined as $p(x, r, \theta, t) \stackrel{\mathcal{F}_{m}}{\longrightarrow}$ $\hat{p}(x, r, t ; m)$.

The subsequent steps in the GP are standard: application of an azimuthal Fourier transform to the equations, followed by a projection on to the POD basis functions $\hat{\boldsymbol{\Phi}}^{(n)}(x, r ; m)^{21}$. Assume that the velocity field is expanded using the first $N_{n, 2}$ POD modes, and azimuthal modes $m=0$ to $m=N_{m}$. Then the resulting set of ODEs can be written as 


$$
\begin{aligned}
& \dot{\hat{\alpha}}^{(n)}(m)=\sum_{j=1}^{N_{n, 2}}\left\{\left(\frac{1}{R e}+\frac{\delta_{n j}}{R e_{T}^{(n)}(m)}\right) L_{n j}(m)+K_{n j}(m)\right\} \hat{\alpha}^{(j)}(m)-\left|\int_{0}^{R} \hat{p}(x, r, t ; m) \hat{\Phi}_{x}^{(n)^{*}}(x, r ; m) r \mathrm{~d} r\right|_{x=X_{1}}^{X_{2}} \\
& +\left(1-\delta_{m 0}\right) \sum_{m^{\prime}=m-N_{m}}^{N_{m}} \sum_{j, l=1}^{N_{n, 2}} Q_{n j l}\left(m, m^{\prime}\right) \times \hat{\alpha}^{(j)}\left(m^{\prime}\right) \hat{\alpha}^{(l)}\left(m-m^{\prime}\right), \forall n \in\left[1, N_{n, 2}\right], \forall m \in\left[0, N_{m}\right] .
\end{aligned}
$$

The expressions for the coefficients appear in the Appendix.

For the assumed incompressible flow under consideration, the pressure term reduces to a surface integral over the boundary of the POD domain ${ }^{35}$. Moreover, owing to the vanishing of velocity fluctuations at the outer radius of the POD domain, the eigenfunctions themselves vanish at this boundary too. Therefore, one obtains the form shown above wherein the pressure needs to be evaluated at the inflow and outflow cross-stream slices only. Even in this simplified form, the term cannot be retained in the ROM since the requisite pressure information cannot be obtained from experiments. In previous applications to similarly unbounded flows ${ }^{35,57}$, this term has been assumed to vanish altogether. Using the DNS database, we verified that indeed no appreciable inaccuracy is introduced by neglecting the pressure term, and this neglect would be implicit for the simulation results presented subsequently. We note here that Noack et al..$^{59}$ showed that for cylinder wake flow simulations, it is most appropriate to retain the pressure term, and to solve for it in parallel using the pressure Poisson equation.

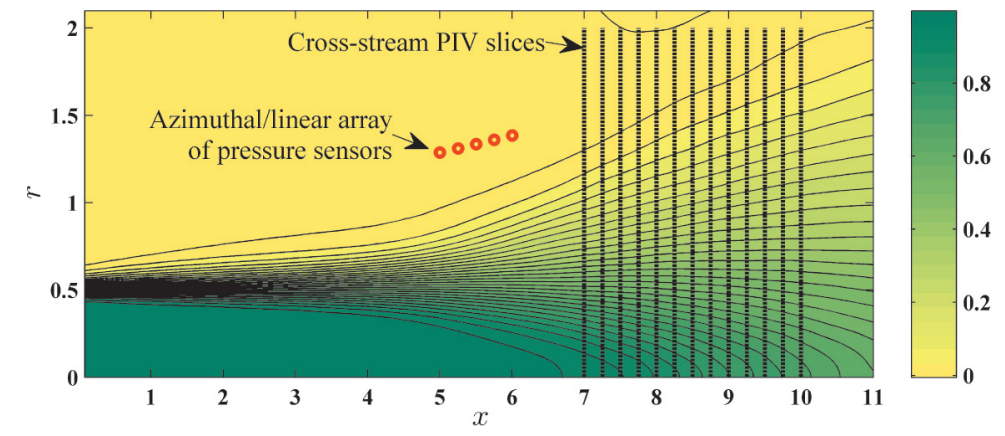

Figure 3. Contour plot of mean axial velocity from the DNS database.

The eigenfunction-basis of the velocity is truncated in both the Fourier space as well as the POD space, keeping only the most energetic modes. The neglected modes have low energy and typically correspond to the smaller-scales of turbulence; this makes them important for dissipation. Since neglecting these modes generally has the effect of making the ROM overly energetic, it is common to model the effect of these neglected modes using an eddy-viscosity representation. Some researchers have incorporated a global eddy viscosity and treated it as a bifurcation parameter ${ }^{31-33}$. Others have computed empirical values of modal eddy viscosities by balancing energy or momentum ${ }^{38,60,61}$. Both these strategies amount in adding linear terms to the ROM, but the latter strategy gives more flexibility. Here, we follow the empirical energy balance route to modeling the eddy viscosity ${ }^{60}$, because it was effective in the cavity tone control model developed in our laboratory ${ }^{38}$. In particular, the non-negative modal eddy Reynolds number $R e_{T}^{(n)}(m)$ appearing in eqn (23) is computed by requiring that the modal kinetic energy be steady in the ensemble-average over the database of realizations. Neglecting the pressure term, this leads to ${ }^{60}$

$$
\begin{aligned}
& \frac{1}{\operatorname{Re}_{T}^{(n)}(m)}=\max \left[0,-\frac{1}{R e}-\frac{1}{L_{n n}(m) \Lambda^{(n)}(m)}\left\{K_{n n}(m) \Lambda^{(n)}(m)+\left(1-\delta_{m 0}\right) \sum_{m=m-N_{m}}^{N_{m}} \sum_{j, l=1}^{N_{n, 2}} Q_{n j l}\left(m, m^{\prime}\right)\right.\right. \\
& \left.\left.\times E\left\{\mathcal{R}\left(\hat{\alpha}^{(n)^{*}}(m) \hat{\alpha}^{(j)}\left(m^{\prime}\right) \hat{\alpha}^{(l)}\left(m-m^{\prime}\right)\right)\right\}\right\}\right], \quad \forall n \in\left[1, N_{n, 2}\right], \quad \forall m \in\left[0, N_{m}\right] .
\end{aligned}
$$




\section{RESULTS AND DISCUSSION}

\subsection{Preliminaries}

The details of the direct numerical simulation database are available in Freund ${ }^{39}$; here we only highlight the most pertinent aspects. The cylindrical computational grid has 80 uniformly spaced azimuthal grid-points. For ease of implementation, the originally non-uniform rectangular $x-r$ grid is linearly interpolated hereby to a uniform square grid with a spacing of 0.0625 (in jet diameter coordinates). All the results were also assessed in the original grid, and no material difference was observed. The data is saved at 2316 consecutive time instants with uniform separation of 0.071 (in $t^{+}$ coordinates).

The ROM is developed for controlling the large-scale structures in the turbulent jet mixing layer near the end of the potential core. If the model domain is too short to accommodate the typical largescale structures in their entirety, then their dynamics cannot be modeled correctly ${ }^{35}$. The constraint at the other extreme is the necessity for the near-field pressure at the upstream location to be wellcorrelated to the velocity field on the cross-stream slice at the most downstream location for the success of the SLSE procedure. The low-Re simulated jet remains laminar for a significant length ${ }^{39}$, whereas the high-Re experimental jet is turbulent at its exit ${ }^{8}$. For the present exercise to be of use in designing later experiments, the modeling should be performed using data from a domain of the simulated jet mixing layer that is turbulent.

A contour plot of the mean axial velocity is shown in Figure 3. The transition from laminar to turbulent flow is difficult to pin-point; however, a difference in the jet-spreading behavior is noted at $x \approx 5$. Practical considerations would prevent the placement of pressure sensors too far downstream in actual applications; this constrains their feasible axial locations. The need to obtain a hydrodynamic signature dictates the radial location of the sensors $30,62,63$. Five conceptual sensors are placed in a uniform linear array from $x=$ 5 to 6 , making an angle of $5.6^{\circ}$ with the jet axis, with the most upstream sensor located at $r=1.29$ (see Figure 3). Alternatively, any or all of them may be replaced by azimuthal arrays of 80 sensors (of the form shown in Figure 1(b)), corresponding to the DNS grid. Following the discussion above, conceptual crossstream slices of the flow were chosen in the range $x=7$ to 10 at intervals of 0.25 to perform the ID slicePOD (see Figure 3). The radial extent of the slice-POD domain was $R=2$.

\subsection{Results of 3D Velocity Field Reconstruction using SLSE}

The application of ID slice-POD to the axisymmetric jet mixing layer is standard, and the results have been published in Tinney et al. ${ }^{29}$. We proceed to a discussion of the accuracy of reconstruction of the 3D velocity field database using SLSE. The details of the implementation of the SLSE, as well as the qualitative nature of the results follow Tinney et al. ${ }^{30}$. We only mention that the finite time Fourier transforms were implemented with $T=28.4 t^{+}$, and overlaps of $21.3 t^{+}$. The cubic spline interpolation is kept out of this exercise for the time-being, to focus on the SLSE alone.

Using any of the methods described previously, one obtains the reconstructed fluctuating velocity field denoted by $\tilde{\mathbf{v}}(r, \theta, t ; x)$. The actual fluctuating velocity field $\mathbf{v}(r, \theta, t ; x)$ at these axial locations is also known for the same time instant $t$. Thus, with the underlying inner product defined as $\left\langle\mathbf{v}^{(1)}, \mathbf{v}^{(2)}\right\rangle:=$ $\int_{0}^{R} \int_{-\pi}^{\pi} \mathbf{v}^{(2) *} \mathbf{v}^{(1)} \mathrm{d} \theta r \mathrm{~d} r$, the following reconstruction error metric is proposed:

$$
e_{\text {recon }}:=\left[\sum_{x \in \mathcal{X}_{v}} E\left\{\|\tilde{\mathbf{v}}(\cdot, \cdot, t ; x)-\mathbf{v}(\cdot, \cdot, t ; x)\|^{2}\right\}\right] /\left[\sum_{x \in \mathcal{X}_{v}} E\left\{\| \mathbf{v}\left(\cdot, \cdot, t ; x \|^{2}\right\}\right] .\right.
$$

The relevant statistics were verified to have converged. Then, the least-squares framework and the orthonormality of the eigenfunction basis afford $e_{\text {recon }}$ to be computed directly from the cross-spectral tensors introduced in Section 2.3. For example, if the complementary SLSE is applied to reconstruct the scalar POD modal coefficients in the ranges $m \in\left[-N_{m r}, N_{m r}\right]$ and $n \in\left[1, N_{n, 1}\right]$ respectively, then we have

$$
e_{\text {recon }} \approx 1-\frac{2 \pi \sum_{x \in \mathcal{X}_{v}} \sum_{i \in\{x, r, \theta\}} \sum_{m=-N_{n r}}^{N_{m r}} \sum_{n=1}^{N_{n, 1}} \int_{f=-\infty}^{\infty} \mathbf{S}_{p \gamma_{i}}^{(n) *}(x, m, f) \mathbf{S}_{p p}^{-1}(m, f) \mathbf{S}_{p \gamma_{i}}^{(n)}(x, m, f) \mathrm{d} f}{\sum_{x \in \mathcal{X}_{v}} E\left\{\|\mathbf{v}(\cdot, \cdot, t ; x)\|^{2}\right\}} .
$$




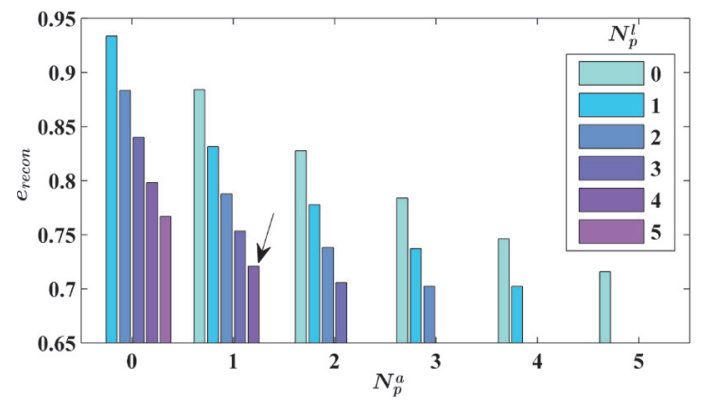

(a)

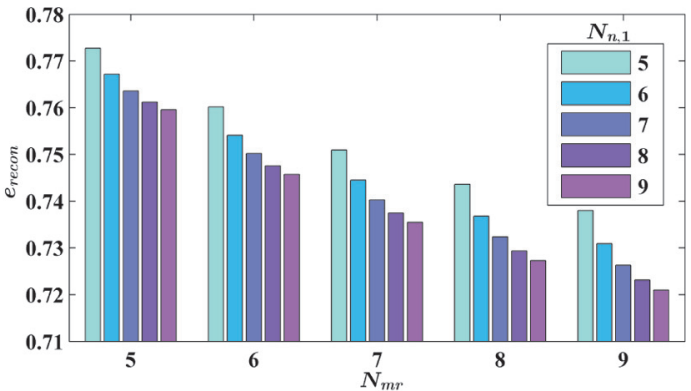

(b)

Figure 4. The reconstruction errors for various parameter choices in stochastic estimation.

In Figure 4(a), all possible pressure sensing configurations are compared using the above metric. In our assay, the sets $\mathcal{X}_{p}^{a}$ and $\mathcal{X}_{p}^{l}$ are mutually exclusive and $0<N_{p}^{a}+N_{p}^{l} \leq 5$; thus only the depicted combinations of $N_{p}^{a}$ and $N_{p}^{l}$ can be evaluated. For each combination, we plot $\left(\arg \min _{\mathcal{X}_{p}^{a}, \chi_{p}^{l}} e_{\text {recon }}\right.$ ) computed from eqn (26) with $N_{m r}=9$ and $N_{n, 1}=9$. Although the errors are quite large in general, it will be shown that the reconstructed database is still useful for the subsequent 2D POD. One observes that beyond the first azimuthal ring array, each additional linear array location provides almost the same informa-tion as an additional azimuthal ring array. Keeping in mind the cost of implementation, we choose the combination with $N_{p}^{a}=1$ and $N_{p}^{l}=4$. Approximately the same $e_{\text {recon }}(=0.72)$ was obtained for all permutations in this case. Henceforth we will exclusively employ the configuration with $\mathcal{X}_{p}^{a}=\{5.5\}$ and $\mathcal{X}_{p}^{l}=\{5,5.25,5.75,6\}$. It is to be noted that this results in a significant improvement over the single azimuthal ring configuration used by Tinney et al. ${ }^{30}$. Figure 4(b) demonstrates the convergence of the error in the $N_{m r}-N_{n, 1}$ space for the chosen configuration.

We have described the application of SLSE to estimate the scalar POD modal coefficients above. Apart from this, SLSE was also applied to the velocity field directly, as well as to the vector POD modal coefficients. The respective values of $e_{\text {recon }}$ for the chosen configuration were 0.69 and 0.75 . Such a performance hierarchy is anticipated since the information provided by the pressure sensors are aggregated to differing degrees in the three schemes. The application of SLSE to the scalar POD modal coefficients, in concert with the cubic spline interpolation, will be used to reconstruct a database of 3D snapshots, and this will be referred to as the "chosen" reconstructed database.

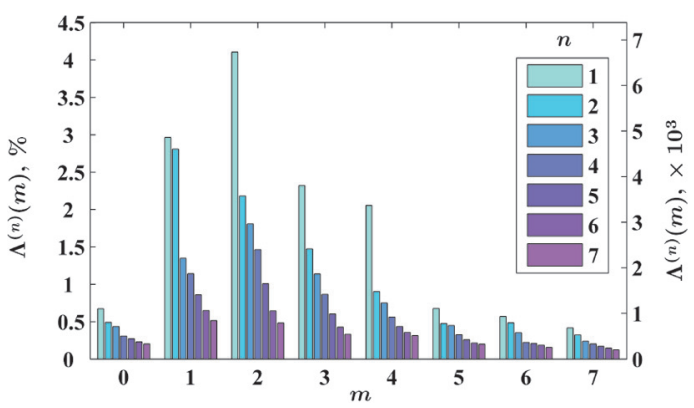

(a)

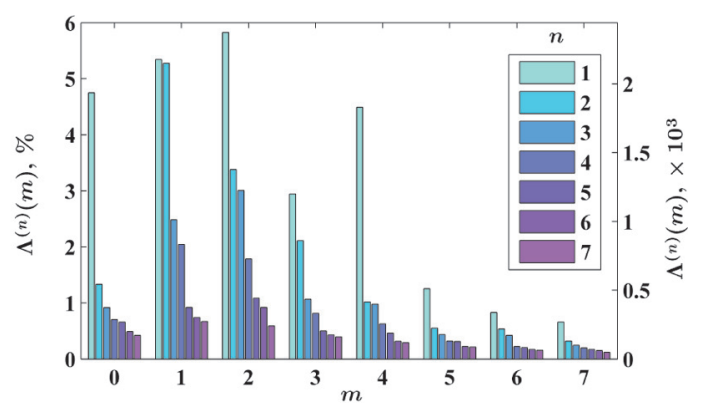

(b)

Figure 5. 2D POD eigenvalue spectra for (a) the original DNS database, and (b) the reconstructed database chosen in Section 3.2. 

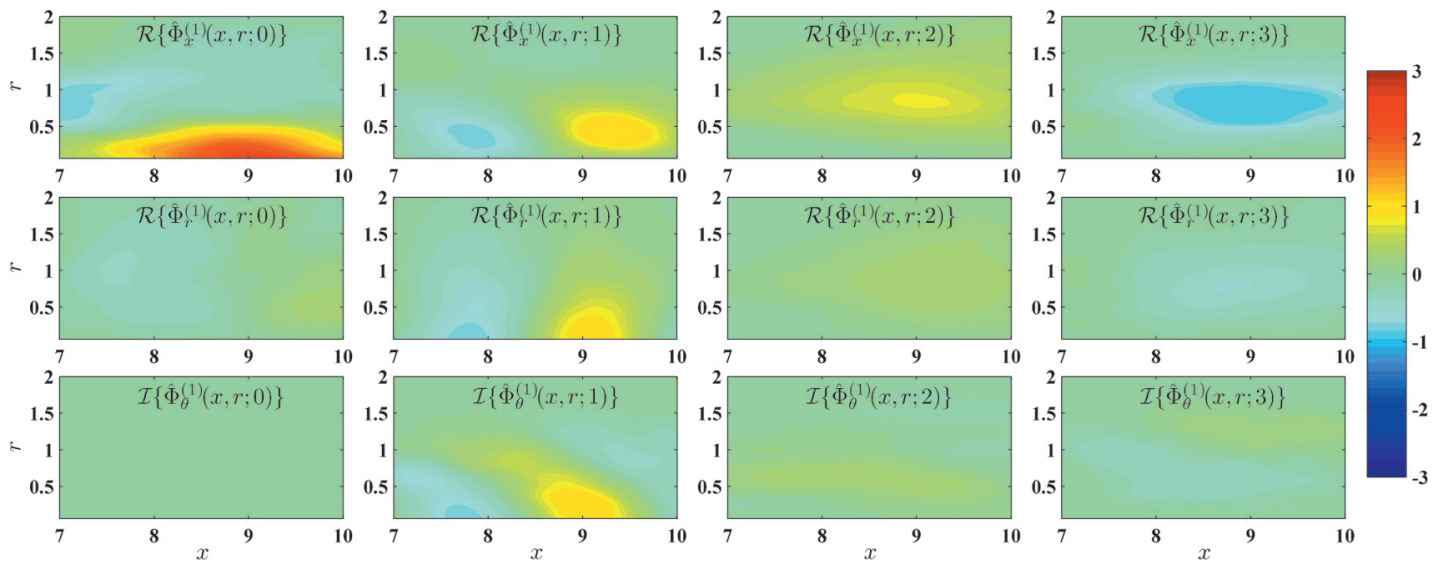

Figure 6. 2D POD eigenfunctions educed from the original DNS database.
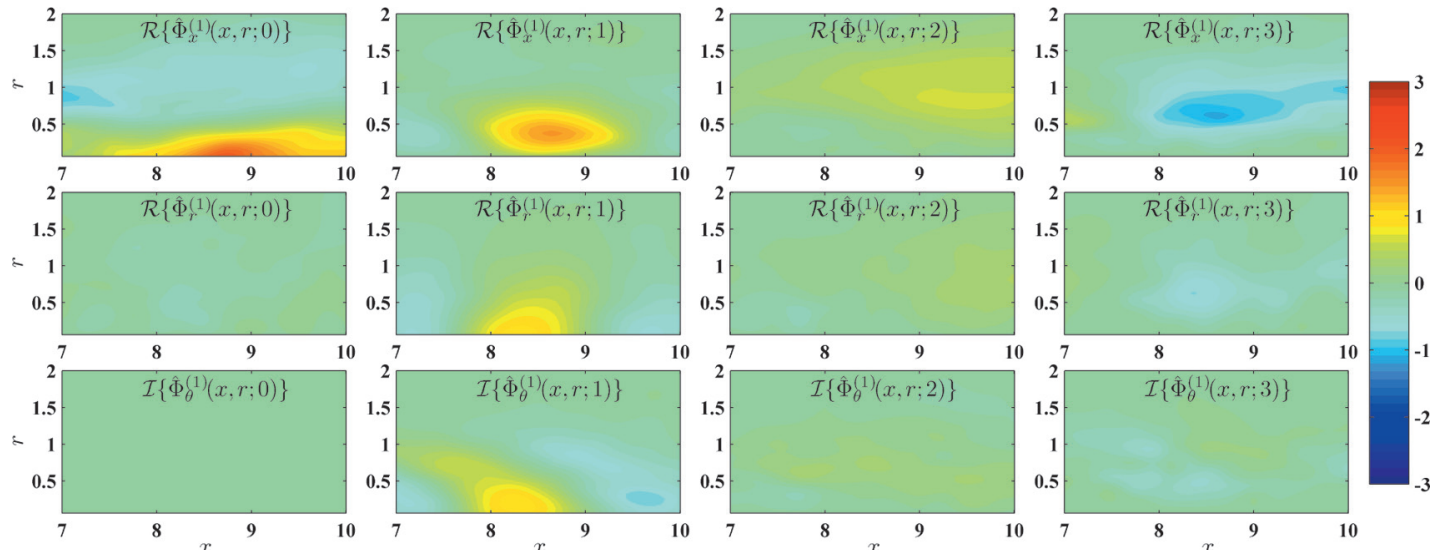

Figure 7. 2D POD eigenfunctions educed from the reconstructed database chosen in Section 3.2.

\subsection{Results of 2D POD on the 3D Velocity Database}

The eigenvalues obtained from the application of the 2D vector POD are presented in Figure 5 as a percentage of the respective total energy captured, as well in terms of their absolute values. For $m \neq 0$, the eigenvalues are doubled to account for the negative azimuthal modes too. Since all the energy is not captured in the reconstructed database, the actual percentages are unimportant, and one should focus on the relative energies within each sub-figure. With this caveat, it is apparent that the reconstruction shows similar trends, although $m=0$ and 4 are over-predicted. The near equality of the 1 st and 2nd POD modes for $m=1$ in both cases is indicative of an approximate periodic behavior in this mode $^{21}$.

Figures 6 and 7 present some representative eigenfunctions corresponding to the two databases depicted in Figure 5. Following the 2D POD counterpart of eqn (10), only the non-trivial components of the first POD eigenfunctions are shown for azimuthal modes 0 through 3 . In spite of the reconstruction errors seen in Figure 4, we note the similarity of the POD eigenfunctions from the original DNS database and the chosen SLSE reconstruction in Figures 6 and 7, respectively. For $m=1$, analysis of Figure 5 indicated a dominant periodic behavior, and the eigenfunctions noticed in the corresponding subparts of Figures 6 and 7 are approximate phase-shifted counterparts of each other. One concludes that while the reconstructed structures are weaker compared to the original, their shapes are captured well by SLSE. Discrepancies in the POD bases would not invalidate the subsequent GP; they would only result in a sub-optimal basis for the expansion, thereby introducing more inaccuracy for the same dimension of the ROM.

If a flow is incompressible, then each of its velocity realizations are solenoidal. The vector POD eigenfunctions for such a flow, being linear combinations of these realizations ${ }^{36}$, inherit the solenoidal 


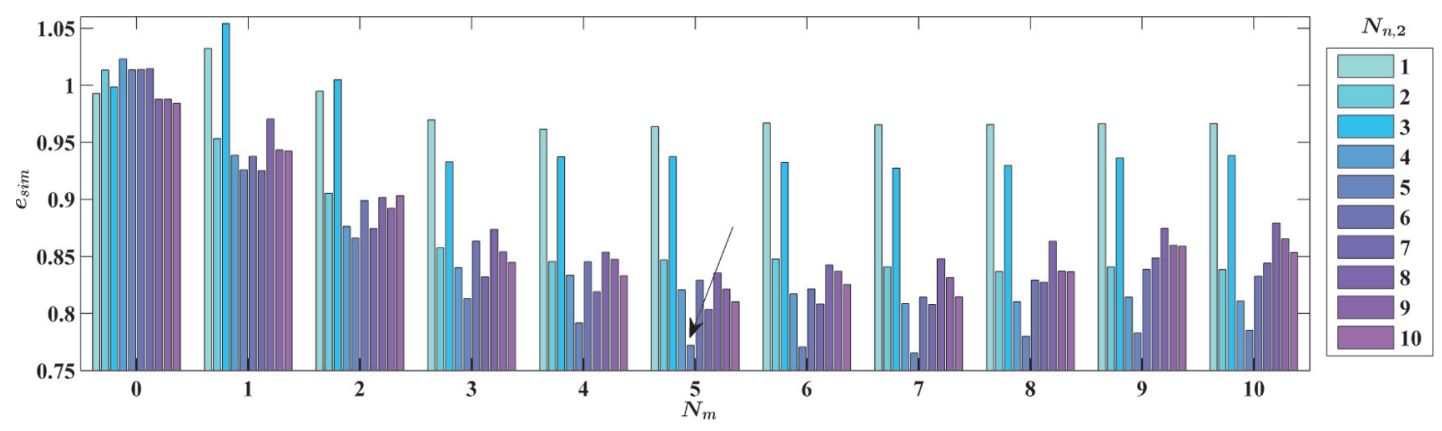

Figure 8. Simulation error for ROMs built from the original DNS database for various choices of the cutoff.

property also. Thus one way of assessing the incompressibility assumption for the present flow is to determine how close $\hat{\boldsymbol{\Phi}}^{(n)}(x, r ; m)$ is to being solenoidal. The following metric

$$
\hat{\boldsymbol{e}}_{\text {sol }}^{(n)}(m):=\frac{\int_{X_{1}}^{X_{2}} \int_{0}^{R}\left|\frac{\partial}{\partial x} \hat{\boldsymbol{\Phi}}_{x}^{(n)}(x, r ; m)+\frac{1}{r} \frac{\partial}{\partial r}\left\{r \hat{\boldsymbol{\Phi}}_{r}^{(n)}(x, r ; m)\right\}+\frac{m}{r} \hat{\boldsymbol{\Phi}}_{\theta}^{(n)}(x, r ; m)\right|^{2} r \mathrm{~d} r \mathrm{~d} x}{\int_{X_{1}}^{X_{2}} \int_{0}^{R}\left[\left|\frac{\partial}{\partial x} \hat{\boldsymbol{\Phi}}_{x}^{(n)}(x, r ; m)\right|^{2}+\left|\frac{1}{r} \frac{\partial}{\partial r}\left\{r \hat{\Phi}_{r}^{(n)}(x, r ; m)\right\}\right|^{2}+\left|\frac{m}{r} \hat{\boldsymbol{\Phi}}_{\theta}^{(n)}(x, r ; m)\right|^{2}\right] r \mathrm{~d} r \mathrm{~d} x} .
$$

is evaluated for the eigenfunctions obtained from the original DNS database with $n \in[1,10]$ and $m \in$ $[0,10]$. The maximum value of $\hat{e}_{\text {sol }}$ is found to be $0.7 \%$, thereby lending support to the incompressibility assumption.

\subsection{Results of Simulation of the ROM Obtained by Galerkin Projection}

The first derivatives of the eigenfunctions and mean velocities appearing in the coefficients of the ROMs (see Appendix) were computed using 6th-order accurate finite differences. The ROMs were simulated using MATLAB's ode45, which is a Runge-Kutta $(4,5)$ ODE solver with automatic step-size selection. Subsequently, the simulation results were linearly interpolated on the time axis of the DNS database for direct comparison. For control-purposes, the ROM needs to predict the flow over short periods, and we focused on a time horizon of $5 t^{+}$in all the results presented here. The initial condition was an arbitrary realization in the middle of the DNS save-record.

Let the simulated fluctuating velocity be denoted by $\tilde{\mathbf{u}}(x, r, \theta, t)$. The actual fluctuating velocity $\mathbf{u}(x, r, \theta, t)$ is also known at the same time instant $t$. Thus, with the underlying inner product defined as $\left\langle\mathbf{u}^{(1)}, \mathbf{u}^{(2)}\right\rangle:=\int_{X_{1}}^{X_{2}} \int_{0}^{R} \int_{-\pi}^{\pi} \mathbf{u}^{(2)^{*}} \mathbf{u}^{(1)} \mathrm{d} \theta r \mathrm{~d} r \mathrm{~d} x$ for any two fields $\mathbf{u}^{(1)}$ and $\mathbf{u}^{(2)}$, we propose the simulation error metric

$$
e_{s i m}:=\frac{E\left\{\|\tilde{\mathbf{u}}(\cdot, \cdot, \cdot, t)-\mathbf{u}(\cdot, \cdot, \cdot, t)\|^{2}\right\}}{E\left\{\|\mathbf{u}(\cdot, \cdot, \cdot, t)\|^{2}\right\}}=1+\frac{2 \pi \sum_{m=-N_{m}}^{N_{m}} \sum_{n=1}^{N_{n, 2}} E\left\{\left|\tilde{\hat{\alpha}}^{(n)}(t ; m)\right|^{2}-2 \mathcal{R}\left(\tilde{\hat{\alpha}}^{(n)^{*}}(t ; m) \hat{\alpha}^{(n)}(t ; m)\right)\right\}}{E\left\{\|\mathbf{u}(\cdot, \cdot, \cdot, t)\|^{2}\right\}}
$$

The second expression follows from the orthonormality of the eigenfunction basis. Here, the POD modal coefficients $\hat{\alpha}^{(n)}(t ; m)$ and $\tilde{\hat{\alpha}}^{(n)}(t ; m)$ correspond to velocities $\mathbf{u}(\cdot, \cdot, \cdot, t)$ and $\tilde{\mathbf{u}}(\cdot, \cdot, \cdot, t)$, respectively.

Figure 8 shows the simulation error evaluated for the ROMs developed using the original DNS database, for various choices of the cutoffs $N_{n, 2}$ and $N_{m}$. The errors are quite large, demonstrating the inherent inaccuracies of low-dimensional modeling for this complex flow. Including the largest number of modes does not always result in the most accurate model $34,36,64$, and this is observed here too. For real-time control, the dimension of the model must be kept at a minimum, and Figure 8 suggests a choice of $N_{n, 2}=5$ and $N_{m}=5$, corresponding to $e_{\text {sim }}=0.77$. These parameters define the 30-dimensional 
Feedback Control of Axisymmetric Jets
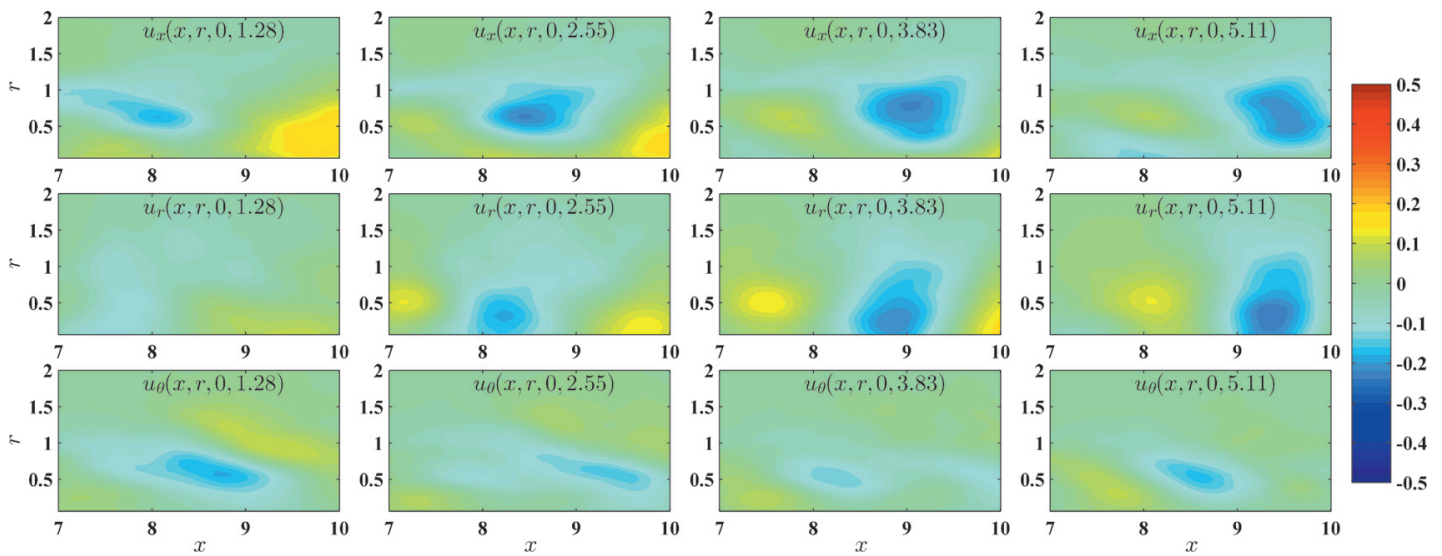

Figure 9. Evolution of a simulated 30D ROM educed from the original DNS database.
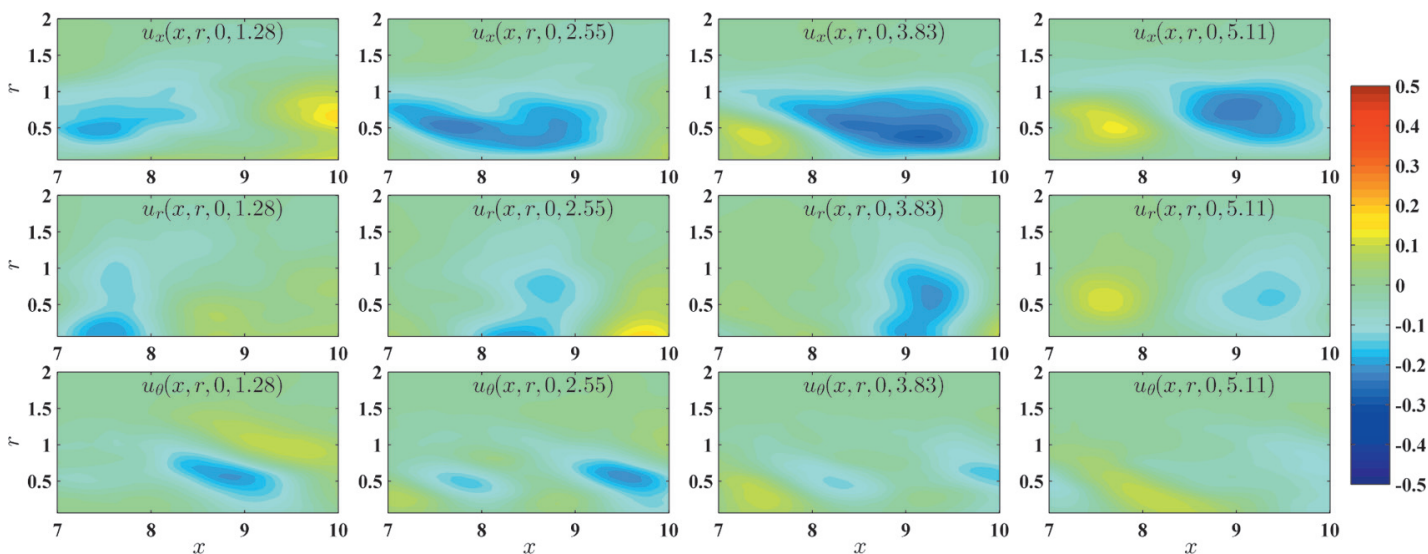

Figure 10. Original DNS realizations projected on the 30D basis used in Figure 9, at matching time instants.
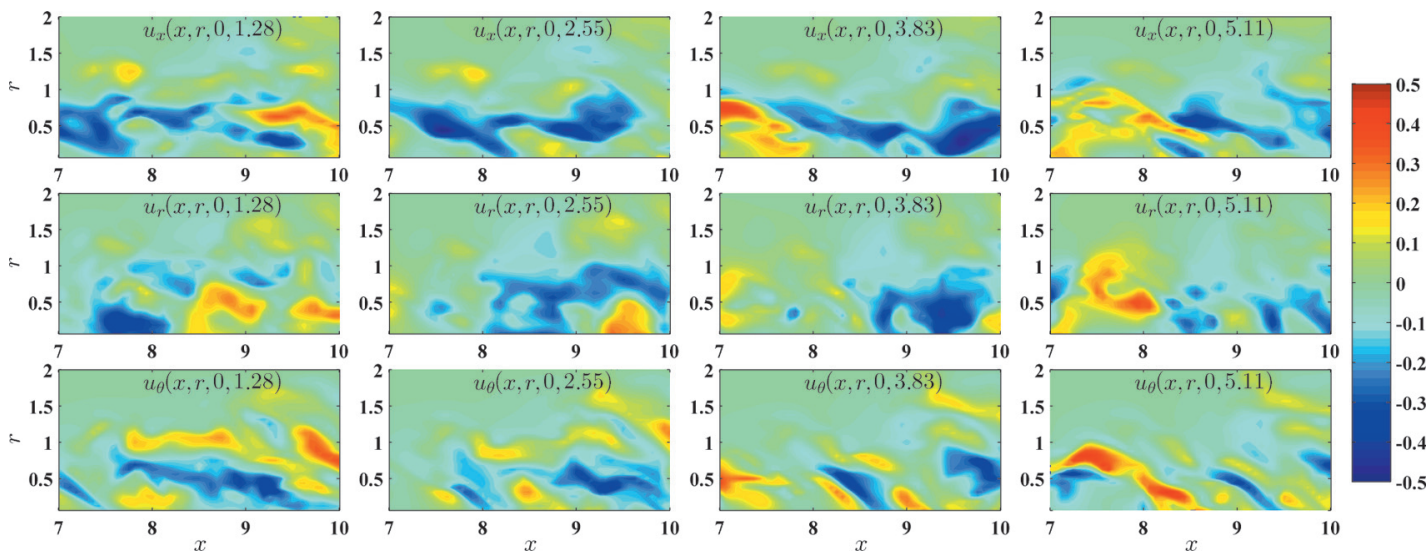

Figure 11. Original DNS realizations at time instants matching Figure 9.

basis that is retained for the ROMs studied hereafter. Note that this basis captures $35 \%$ of the total energy of the flow.

The simulation error $e_{\text {sim }}$ is a gross metric; to get a better intuition, we present some snapshots of the flow evolution from the ROM simulation in Figure 9. The velocity fluctuations on the $\theta=0$ plane are shown. The time instants are measured from the initial condition chosen for generating Figure 8. Figure 9 is to be compared with Figure 10, which shows the projection of the corresponding realizations 

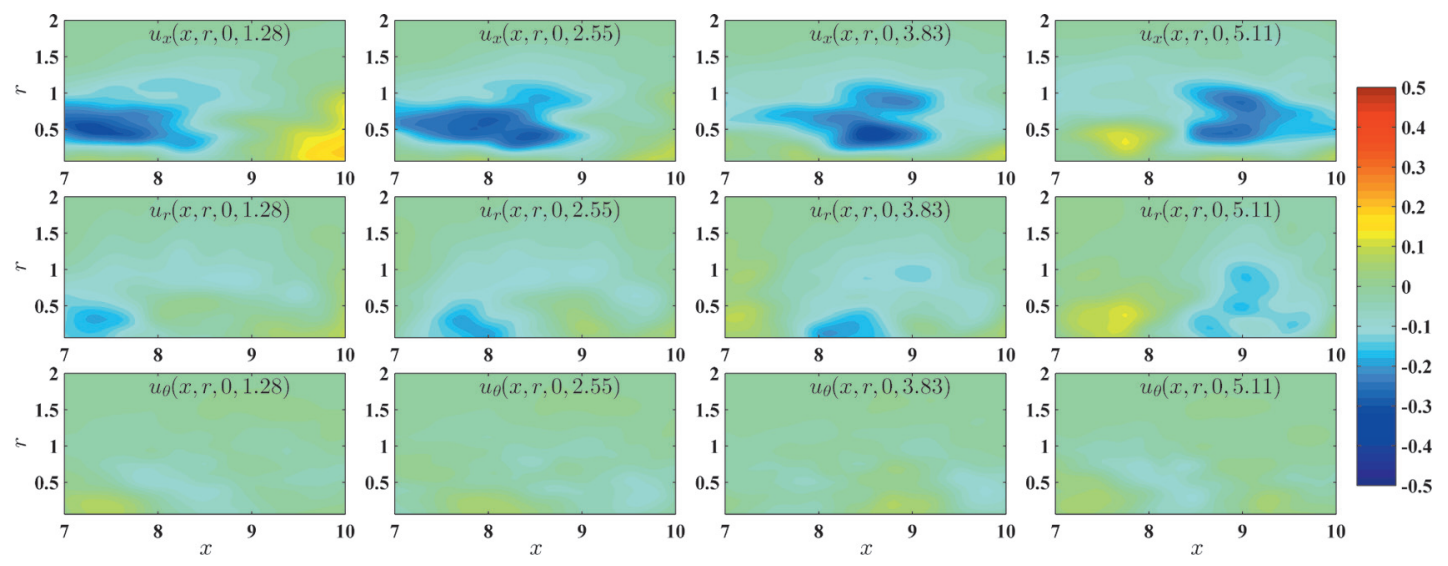

Figure 12. Evolution of a simulated 30D ROM educed from the reconstructed database chosen in Section 3.2.
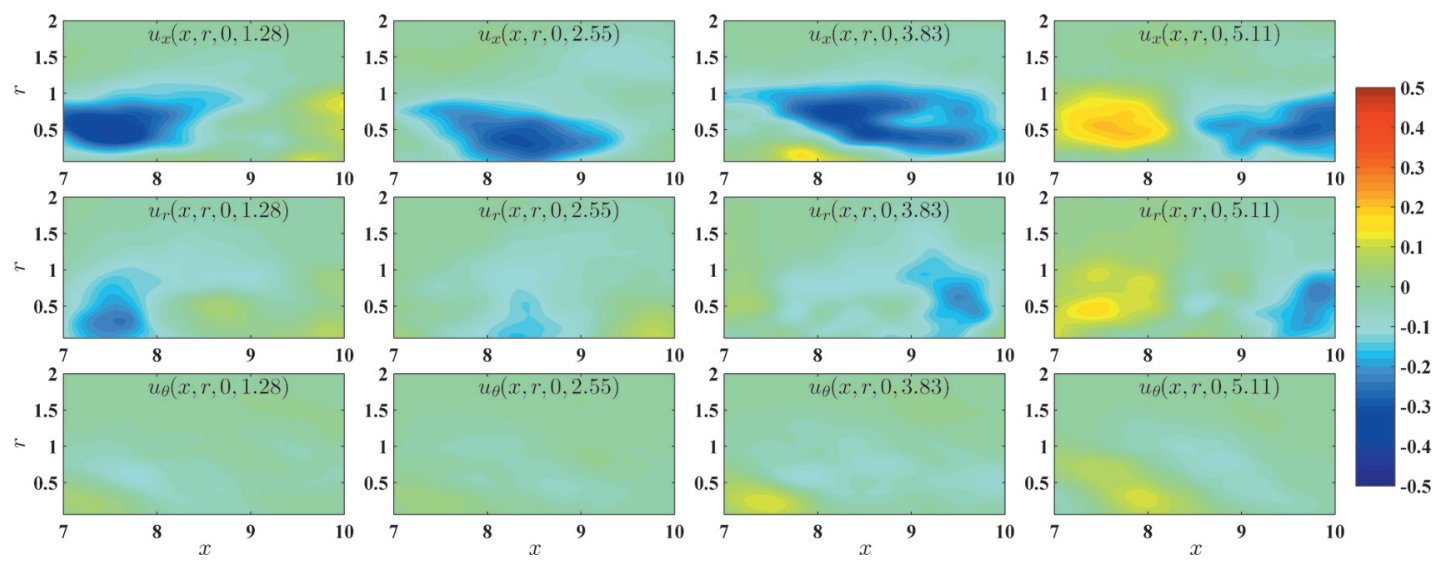

Figure 13. Reconstructed realizations projected on the 30D basis used in Figure 12, at matching time instants.

of the original DNS database onto the same 30D eigenfunction basis. In Figure 11 we also present the actual snapshots at the corresponding instants. As expected, the overall discrepancies are quite large, but most of the differences result from the use of a partial basis for the reconstruction. The different components of velocity are modeled with different accuracies with decreasing order being axial, radial, and azimuthal. The turbulent kinetic energies associated separately with the three components were also found to be ordered similarly for this database. Since the underlying vector POD is weighted towards the more energetic component, the perceived difference in modeling accuracy is to be expected. Overall, the low-dimensional dynamics of the flow are seen to be captured with good accuracy over the selected simulation time horizon.

The error metric defined in eqn (28) essentially compares the realizations represented partially in Figure 9 with those in Figure 11. One can also compare Figure 9 with Figure 10, i.e., compare the simulated realizations from a ROM to the actual realizations projected onto the underlying basis for the ROM. This gave an error value of 0.45 for the 30D ROM under consideration. This demonstrates that a large part of the simulation error defined in eqn (28) can be explained by the relatively low percentage of energy captured by the 30D basis. The remaining part of the error is due to the inability of this basis to capture the dynamics of the retained modes (the Galerkin projection error). However, the analysis of Figure 8 makes it clear that the 30D basis is still optimal keeping in mind the requirement that the control-oriented model should have a very low dimension.

An ROM was also derived by Galerkin projection onto the 2D POD basis educed from the reconstructed database chosen in Section 3.2. The cutoffs were retained as $N_{n, 2}=5$ and $N_{m}=5$ to define a new 30D basis. The initial time matched the previous simulation, and the corresponding reconstructed realization was projected onto the new 30D basis to obtain the set of initial POD modal coefficients. 
The evolution of this ROM is shown in Figure 12; for this model $e_{\text {sim }}=0.91$. For comparison, Figure 13 shows the projection of the corresponding reconstructed realizations onto the same basis. The shape and strength of the structures are seen to be quite alike in these two figure, thereby once again attesting to the fidelity of the Galerkin projection. If, as in the above analysis, the simulated field is compared to the projected field, then the error value was 0.69 .

The availability of the DNS database allowed us to evaluate two additional models that incorporated compressibility effects. The first one employed the approach of Rowley et al. ${ }^{58}$, who proposed an isentropic assumption and chose the state vector to include the local speed of sound in addition to the components of velocity. The same authors introduced an inner product based on the stagnation enthalpy. The eddy viscosity model described in Section 2.5 was replicated to incorporate the dissipation necessary to counteract the truncation of the basis. This method was used to derive a 30D ROM from the original DNS database. The simulation error was computed in a manner akin to eqn (28), and was found to be 0.82 . The other modeling technique was adapted from the works of Iollo et al. ${ }^{65}$, Gloerfelt ${ }^{66}$ who projected the full compressible Navier-Stokes equations using a state vector consisting of the specific volume and pressure in addition to the velocity components. In the absence of a suitable energy-related inner product, they simply extended the inner product definition from the incompressible case; this makes the POD and GP dependent on the particular normalization used. Following their work, we normalized the specific volume by that of the ambient flow, and the pressure as in Section 2.1. The eddy viscosity modeling was repeated. A 30D ROM built in this manner demonstrated a simulation error of 0.78 . In fact, both the models demonstrated simulation error plots quite similar to Figure 8. This substantiates the claim that the neglect of the compressibility effects of the Mach 0.9 flow is not the source of the simulation errors; instead the source is to be found in the slow convergence of the POD eigenspectrum.

The related work of Schlegel et al. ${ }^{67}$ came to our attention at the galley proof stage of publication. The particular contribution of our research is the proposed modeling strategy that can be adapted for experimental implementation in high Reynolds number flows.

\section{Conclusion}

The first step in model-based feedback flow control is the development of a reduced-order model of the unforced flow; this has been pursued here for an axisymmetric jet. An existing direct numerical simulation database of a Mach 0.9 low Reynolds number jet is used to guide the modeling, with an eye toward feasibility of later experimental implementation. The two phases of the modeling are (a) reconstructing a database of snapshots of the 3-component velocity field over the pertinent 3D domain of the jet mixing layer from experimentally accessible measurements, and $(b)$ determining the dynamics of the most energetic structures in this domain.

For the first phase, we adopted the spectral linear stochastic estimation technique presented by Tinney et al. ${ }^{29,30}$ that employs pressure measurements on an azimuthal array in the irrotational nearfield of the jet. The estimation coefficients are determined from the cross-spectra between pressure measurements and low-dimensional representations of the velocity fields on individual cross-stream slices covering the axial domain of interest in the jet mixing layer. We showed here that the reconstruction fidelity is substantially improved by incorporating an additional linear array of pressure sensors in the near-field, at little extra cost. The statistical significance of the estimation was improved by systematically enforcing the axisymmetry axiom for the flow.

For the second phase, proper orthogonal decomposition was used to educe an orthonormal partial basis for the velocity field consisting of the most energetic structures. Subsequently, Galerkin projection of the incompressible Navier-Stokes equations onto this basis yielded a set of ordinary differential equations that govern their dynamics. To validate the procedure, a 30-dimensional basis was first derived from the original database. Simulations of the resulting model demonstrated that the evolution of the large-scale structures are well-captured. Finally, a basis of the same dimension was obtained from the database reconstructed in the first phase. Simulations of the resulting ROM showed that it may be acceptable for the purposes of feedback control.

The continuation of this research would include physical experiments to obtain the empirical database for modeling purposes, as well as the explicit inclusion of the effect of actuation. A promising approach is to model the downstream effect of actuation as weak compression waves that can be introduced in the model through the pressure term in eqn (23). Further work would involve the design of observers that would use measurements of the near-field pressure to estimate the mixing effect in the 
near-field shear layer and the far-field noise. Once all these pieces are in place, the feedback control algorithm may be developed. In all this, it must be clear that the modeling of the unforced flow pursued here constitutes an important first step towards the end goal.

\section{ACKNOWLEDGMENTS}

The research was supported in part by the Air Force Office of Scientific Research through a joint research program with Syracuse University and Clarkson University, with Dr. John Schmisseur as the program manager; this is greatly appreciated. The authors would also like to thank Dr. J. B. Freund for providing the DNS database, Dr. Edgar Caraballo for insightful discussions, and Dr. Mark Glauser for the collaboration.

\section{APPENDIX: COEFFICIENTS OF THE ROM}

The expressions for the coefficients of the ROM presented in eqn (23) are

$$
\begin{aligned}
& L_{n j}(m)=\int_{0}^{R}\left[\left|\frac{\partial \hat{\Phi}_{i}^{(j)}(m)}{\partial x} \hat{\boldsymbol{\Phi}}_{i}^{(n)^{*}}(m)\right|_{X_{1}}^{X_{2}}-\int_{X_{1}}^{X_{2}} \frac{\partial \hat{\Phi}_{i}^{(j)}(m)}{\partial x} \frac{\partial \hat{\Phi}_{i}^{(n)^{*}}(m)}{\partial x} \mathrm{~d} x\right] r \mathrm{~d} r \\
& +\int_{X_{1}}^{X_{2}}\left[\left|r \frac{\partial \hat{\Phi}_{i}^{(j)}(m)}{\partial r} \hat{\Phi}_{i}^{(n)^{*}}(m)\right|_{0}^{R}-\int_{0}^{R} \frac{\partial \hat{\Phi}_{i}^{(j)}(m)}{\partial r} \frac{\partial \hat{\Phi}_{i}^{(n)^{*}}(m)}{\partial r} r \mathrm{~d} r\right] \mathrm{d} x \\
& +\int_{X_{1}}^{X_{2}} \int_{0}^{R}\left[-m^{2} \hat{\boldsymbol{\Phi}}_{i}^{(j)}(m)-\left\{\hat{\boldsymbol{\Phi}}_{r}^{(j)}(m)+2 \mathrm{i} m \hat{\Phi}_{\theta}^{(j)}(m)\right\} \boldsymbol{\delta}_{i r}-\left\{\hat{\boldsymbol{\Phi}}_{\theta}^{(j)}(m)-2 \mathrm{i} m \hat{\boldsymbol{\Phi}}_{r}^{(j)}(m)\right\} \boldsymbol{\delta}_{i \theta}\right] \hat{\boldsymbol{\Phi}}_{i}^{(n)^{*}}(m) \frac{\mathrm{d} r \mathrm{~d} x}{r} \\
& K_{n j}(m)=\int_{X_{1}}^{X_{2}} \int_{0}^{R}\left[-\bar{U}_{x} \frac{\partial \hat{\Phi}_{i}^{(j)}(m)}{\partial x}-\bar{U}_{r} \frac{\partial \hat{\Phi}_{i}^{(j)}(m)}{\partial r}-\hat{\Phi}_{x}^{(j)}(m) \frac{\partial \bar{U}_{x}}{\partial x} \delta_{i x}-\hat{\Phi}_{r}^{(j)}(m) \frac{\partial \bar{U}_{x}}{\partial r} \delta_{i x}\right. \\
& \left.-\hat{\boldsymbol{\Phi}}_{x}^{(j)}(m) \frac{\partial \bar{U}_{r}}{\partial x} \delta_{i r}-\hat{\boldsymbol{\Phi}}_{r}^{(j)}(m) \frac{\partial \bar{U}_{r}}{\partial r} \delta_{i r}-\frac{\bar{U}_{r} \hat{\Phi}_{\theta}^{(j)}(m)}{r} \delta_{i \theta}\right] \hat{\boldsymbol{\Phi}}_{i}^{(n)^{*}}(m) r \mathrm{~d} r \mathrm{~d} x \\
& Q_{n j l}\left(m, m^{\prime}\right)=\int_{X_{1}}^{X_{2}} \int_{0}^{R}\left[-\left\{\hat{\boldsymbol{\Phi}}_{x}^{(j)}\left(m^{\prime}\right) \frac{\partial}{\partial x}+\hat{\boldsymbol{\Phi}}_{r}^{(j)}\left(m^{\prime}\right) \frac{\partial}{\partial r}+\frac{\mathrm{i}\left(m-m^{\prime}\right) \hat{\boldsymbol{\Phi}}_{\theta}^{(j)}\left(m^{\prime}\right)}{r}\right\}\right] \hat{\boldsymbol{\Phi}}_{i}^{(l)}\left(m-m^{\prime}\right) \\
& \left.+\frac{\hat{\Phi}_{\theta}^{(j)}\left(m^{\prime}\right) \delta_{i r}-\hat{\Phi}_{r}^{(j)}\left(m^{\prime}\right) \delta_{i \theta}}{r} \hat{\Phi}_{\theta}^{(l)}\left(m-m^{\prime}\right)\right] \hat{\Phi}_{i}^{(n)^{*}}(m) r \mathrm{~d} r \mathrm{~d} x .
\end{aligned}
$$

The dependence of the eigenfunctions on $x$ and $r$ have been suppressed for notational convenience. For accuracy of numerical differentiation, the second derivatives of the eigenfunctions have been transformed into first derivatives by integration-by-parts. The symmetries of the POD eigenfunctions established in the 2D POD counterpart of eqn (10) immediately lead to the conclusion that all the coefficients are purely real.

\section{REFERENCES}

[1] P. Jordan and Y. Gervais. Subsonic jet aeroacoustics: associating experiment, modelling and simulation. Experiments in Fluids, 44(1):1-21, 2008.

[2] E.J. Gutmark, K.C. Schadow, and K.H. Yu. Mixing enhancement in supersonic free shear flows. Annu. Rev. Fluid Mech., 27(1):375-417, 1995.

[3] Y.G. Utkin, S. Keshav, J.-H. Kim, J. Kastner, I.V. Adamovich, and M. Samimy. Development and use of localized arc filament plasma actuators for high-speed flow control. Journal of Physics D: 
Applied Physics, 40(3):685-694, 2007.

[4] M. Samimy, J.-H. Kim, J. Kastner, I. Adamovich, and Y. Utkin. Active control of high-speed and highReynolds-number jets using plasma actuators. Journal of Fluid Mechanics, 578:305-330, 2007.

[5] J.-H. Kim, J. Kastner, and M. Samimy. Active control of a high Reynolds number Mach 0.9 axisymmetric jet. AIAA Journal, 47(1):116-128, 2009.

[6] M. Samimy, J.-H. Kim, J. Kastner, I. Adamovich, and Y. Utkin. Active control of a Mach 0.9 jet for noise mitigation using plasma actuators. AIAA Journal, 45(4):890-901, 2007.

[7] J. Kastner, J.-H. Kim, and M. Samimy. A study of the correlation of large-scale structure dynamics and far-field radiated noise in an excited Mach 0.9 jet. International Journal of Aeroacoustics, 8(3):231-259, 2009.

[8] M. Kearney-Fischer, J.-H. Kim, and M. Samimy. Control of a high Reynolds number Mach 0.9 heated jet using plasma actuators. Physics of Fluids, 21:95-101, 2009.

[9] M. Kearney-Fischer, J.-H. Kim, and M. Samimy. Noise control of a high Reynolds number Mach 0.9 heated jet using plasma actuators. In 15th AlAA/CEAS Aeroacoustics Conference, AIAA Paper 2009-3188, 2009.

[10] A. Sinha, K. Kim, J.-H. Kim, A. Serrani, and M. Samimy. Extremizing feedback control of a highspeed and high Reynolds number jet. AIAA Journal, 48(2):387-399, 2010.

[11] S.S. Collis, R.D. Joslin, A. Seifert, and V. Theofilis. Issues in active flow control: theory, control, simulation, and experiment. Prog. Aerosp. Sci., 40:237-289, 2004.

[12] E. Caraballo, C. Kasnakoglu, A. Serrani, and M. Samimy. Control input separation methods for reduced-order model-based feedback flow control. AIAA Journal, 46(9):2306-2322, 2008.

[13] M. Pastoor, L. Henning, B.R. Noack, R. King, and G. Tadmor. Feedback shear layer control for bluff body drag reduction. Journal of Fluid Mechanics, 608:161-196, 2008.

[14] A.A. Tchieu, A.T. Kutay, J.A. Muse, A.J. Calise, and A. Leonard. Validation of a low-order model for closed-loop flow control enabled flight. In 4th AIAA Flow Control Conference, AIAA Paper 2008-3863, 2008.

[15] B.R. Noack, K. Afanasiev, M. Morzynski, G. Tadmor, and F. Thiele. A hierarchy of lowdimensional models for the transient and post-transient cylinder wake. Journal of Fluid, Mechanics, 497:335-363, 2003.

[16] D.M. Luchtenburg, B. Gunther, B. Noack, R. King, and G. Tadmor. A generalized mean-field model of the natural and high-frequency actuated flow around a high-lift configuration. Journal of Fluid, Mechanics, 623:283-316, 2009.

[17] L. Perret, E. Collin, and J. Delville. Polynomial identification of POD based low-order dynamical system. Journal of Turbulence, 7(17):1-15, 2006.

[18] L. Henning, M. Pastoor, B.R. Noack, R. King, and G. Tadmor. Feedback control applied to the bluff body wake. In R. King, editor, Active Flow Control, pages 369-390. Springer-Verlag, 2007.

[19] M.O. Efe, M. Debiasi, P. Yan, H. Ozbay, and M. Samimy. Neural network-based modelling of subsonic cavity flows. International Journal of Systems Science, 39(2):105-117, 2008.

[20] S. Siegel, J. Seidel, C. Fagley, D.M. Luchtenburg, K. Cohen, and T. McLaughlin. Low-dimensional modelling of a transient cylinder wake using double proper orthogonal decomposition. Journal of Fluid Mechanics, 610:1-42, 2008.

[21] P. Holmes, J. Lumley, and G. Berkooz. Turbulence, Coherent Structures, Dynamical Systems and Symmetry. Cambridge University Press, 1996.

[22] M. Marion and R. Temam. Nonlinear Galerkin methods. SIAM J. Numer. Anal, 2005(5):1139-1157, 1989.

[23] A. Debussche, T. Dubois, and R. Temam. The nonlinear Galerkin method: A multiscale method applied to the simulation of homogeneous turbulent flows. Theoret Comput. Fluid, Dynamics, 7:279-315, 1995.

[24] J.L. Lumley. The structure of inhomogeneous turbulent flows. In A.M. Yaglom and V.I. Tatarsky, editors, Atm. Turb. and Radio Wave Prop., pages 166-178. Nauka, Moscow, 1967.

[25] M.N. Glauser, S.J. Leib, and W.K. George. Coherent structures in the axisymmetric jet mixing layer. In Proc. Fifth Int. Symp. Turbulent Shear Flows, Ithaca, USA, 1985. 
[26] S. Gamard, W.K. George, D. Jung, and S. Woodward. Application of a slice proper orthogonal decomposition to the far field of an axisymmetric turbulent jet. Physics of Fluids, 14(7):2515-2522, 2002.

[27] D.H. Jung, S. Gamard, and W.K. George. Downstream evolution of the most energetic modes in a turbulent axisymmetric jet at high Reynolds number. Part 1. The near-field region. Journal of Fluid Mechanics, 514:173-204, 2004.

[28] M.O. Iqbal and F. O. Thomas. Coherent structure in a turbulent jet via a vector implementation of the proper orthogonal decomposition. Journal of Fluid Mechanics, 571:281-326, 2007.

[29] C.E. Tinney, M.N. Glauser, and L.S. Ukeiley. Low-dimensional characteristics of a transonic jet. Part 1. Proper orthogonal decomposition. Journal of Fluid Mechanics, 612:107-141, 2008.

[30] C.E. Tinney, L.S. Ukeiley, and M.N. Glauser. Low-dimensional characteristics of a transonic jet. Part 2. Estimate and far-field prediction. Journal of Fluid Mechanics, 615:53-92, 2008.

[31] N. Aubry, P. Holmes, J.L. Lumley, and E. Stone. The dynamics of coherent structures in the wall region of a turbulent boundary layer. Journal of Fluid Mechanics, 192:115-173, 1988.

[32] X. Zheng. A low dimensional description of the axisymnietric jet mixing layer. $\mathrm{PhD}$ thesis, Clarkson University, 1990.

[33] L.S. Ukeiley, L. Cordier, R. Manceau, J. Delville, M.N. Glauser, and J.-P. Bonnet. Examination of large-scale structures in a turbulent plane mixing layer. Part 2. Dynamical systems model. Journal of Fluid Mechanics, 441:67-108, 2001.

[34] D. Rempfer. On low-dimensional Galerkin models for fluid flow. Theoret. Comput. Fluid Dynamics, 14:75-88, 2000.

[35] M. Rajaee, S.K.F. Karlsson, and L. Sirovich. Low-dimensional description of free-shear-flow coherent structures and their dynamical behaviour. Journal of Fluid Mechanics, 258:1-29, 1994.

[36] C.R. Rowley. Modeling, Simulation, and Control of Cavity Flow Oscillations. PhD thesis, California Institute of Technology, 2002.

[37] M. Samimy, M. Debiasi, E. Caraballo, A. Serrani, X. Yuan, J. Little, and J.H. Myatt. Feedback control of subsonic cavity flows using reduced-order models. Journal of Fluid Mechanics, 579:315-346, 2007.

[38] E. Caraballo, J. Little, M. Debiasi, and M. Samimy. Development and implementation of an experimental-based reduced-order model for feedback control of subsonic cavity flows. ASME J. Fluids Enrng., 129(7):813-824, 2007.

[39] J.B. Freund. Noise sources in a low-Reynolds-number turbulent jet at Mach 0.9. Journal of Fluid Mechanics, 438(1):277-305, 2001.

[40] J. Kastner, M. Samimy, J. Hileman, and J.B. Freund. Comparison of noise mechanisms in high and low Reynolds number high-speed jets. AIAA Journal, 44(10):2251-2258, 2006.

[41] J. Kim and T.R. Bewley. A linear systems approach to flow control. Annu. Rev. Fluid Mech., 39:383-417, 2007.

[42] L. Sirovich. Turbulence and the dynamics of coherent structures, Parts I-III. Q. Appl. Math., XLV(3):561-590, 1987.

[43] W.K. George. Insight into the dynamics of coherent structures from a proper orthogonal decomposition. In Proc. Symp. on Near Wall Turbulence, Dubrovnik, Yugoslavia, 1988.

[44] J.S. Bendat and A.G. Piersol. Engineering Applications of Correlation and Spectral Analysis. John Wiley, 3rd edition, 2000.

[45] P. Moin and R.D. Moser. Characteristic-eddy decomposition of turbulence in a channel. Journal of Fluid Mechanics, 200: 471-509, 1989.

[46] R.J. Adrian. On the role of conditional averages in turbulence theory. In Turbulence in Liquids; Proc. 4th Biennal Symp. Missouri, USA (A77-40426 18-34), Princeton, NJ, pages 323-332. Science Press, 1977.

[47] R.J. Adrian. Stochastic estimation of conditional structure: a review. Applied, Scientific Research, 53:291-303, 1994. 
[48] J.-P. Bonnet, D.R. Cole, J. Delville, M.N. Glauser, and L.S. Ukeiley. Stochastic estimation and proper orthogonal decomposition: Complementary techniques for identifying structure. Experiments in Fluids, 17(5):307-314, 1994.

[49] J. Boree. Extended proper orthogonal decomposition: A tool to analyse correlated events in turbulent flows. Experiments in Fluids, 35(2):188-192, 2003. ISSN 07234864.

[50] J.A. Taylor and M.N. Glauser. Towards practical flow sensing and control via POD and LSE based low-dimensional tools. ASME J. Fluids Enrng., 126(3):337-345, 2004.

[51] D. Ewing and J.H. Citriniti. Examination of a LSE/POD complementary technique using single and multi-time information in the axisymnietric shear layer. In J.N. Sorensen, E.J. Hopfinger, and N. Aubry, editors, Proceedings of the IUTAM Symposium on simulation and identification of organized structures in flows, Lyngby, Denmark, 25-29 May 1997, pages 375-384. Kluwer Academic Press, 1999.

[52] C.E. Tinney, F. Coiffet, J. Delville, A.M. Hall, P. Jordan, and M.N. Glauser. On spectral linear stochastic estimation. Experiments in Fluids, 41(5):763-775, 2006.

[53] C.E. Tinney, P. Jordan, A.M. Hall, J. Delville, and M.N. Glauser. A time-resolved estimate of the turbulence and sound source mechanisms in a subsonic jet flow. Journal of Turbulence, 8(7):1-20, 2007.

[54] C. DeBoor. A Practical Guide to Splines. Springer-Verlag, New York, 1978.

[55] H. Schlichting. Boundary-Layer Theory. McGraw Hill, 1968.

[56] A.E. Deane, I.G. Kevrekidis, S.C. Karniadakis, and G.E. Orszag. Low-dimensional models for complex geometry flows: Application to grooved channels and circular cylinders. Physics of Fluids A, 3(10):2337-2354, 1991.

[57] D.Rempfer and H.F. Fasel. Dynamics of three-dimensional coherent structures in a flat-plate boundary layer. Journal of Fluid Mechanics, 275:257-283, 1994.

[58] C.R. Rowley, T. Colonius, and R.M. Murray. Model reduction for compressible flows using POD and Galerkin projection. Physica D, 189:115-129, 2004.

[59] B.R. Noack, P. Papas, and P. Monkewitz. The need for a pressure-term representation in empirical Galerkin models of incompressible shear flows. Journal of Fluid Mechanics, 523:339-365, 2005.

[60] W. Cazemier, R.W.C.P. Verstappen, and A.E.P. Veldman. Proper orthogonal decomposition and low-dimensional models for driven cavity flows. Physics of Fluids, 10(7):1685-1699, 1998.

[61] M. Couplet, P. Sagaut, and C. Basdevant. Intermodal energy transfers in a proper orthogonal decomposition-Galerkin representation of a turbulent separated flow. Journal of Fluid Mechanics, 491:275-284, 2003.

[62] R.E.A. Arndt, D.F. Long, and M.N. Glauser. The proper orthogonal decomposition of pressure fluctuations surrounding a turbulent jet. Journal of Fluid Mechanics, 340(1): 1-33, 1997.

[63] F. Coiffet, P. Jordan, J. Delville, Y. Gervais, and F. Ricaud. Coherent structures in subsonic jets: a quasi-irrotational source mechanism? International Journal of Aeroacoustics, 5(1):67-89, 2006.

[64] E. Caraballo, M. Samimy, and J. DeBonis. Low dimensional modeling of flow for closed-loop flow control. In 41st AIAA Aerospace Sciences Meeting and Exhibit, AIAA Paper 2003-0059, 2003.

[65] A. Iollo, S. Lanteri, and J.-A. Desideri. Stability properties of POD-Galerkin approximations for the compressible navier-stokes equations. Theoret. Cornput. Fluid Dynamics, 13:377-396, 2000.

[66] X. Gloerfelt. Compressible proper orthogonal decomposition/Galerkin reduced-order model of self-sustained oscillations in a cavity. Physics of Fluids, 20:115105, 2008.

[67] M. Schlegel, B.R. Noack, P. Comte, D. Kolomenskiy, K. Schneider, M. Farge, D.M. Luchtenburg, J.E. Scouten, and G.Tadmor. Reduced-order modelling of turbulent jets for noise control. In C. Brun et al., editors, Num. Sim. of Turbulent Flows \& Noise Generation, NNFM 104, pages 3-27. Springer, 2009. 